\title{
Economic Model Predictive Control for Time-Varying System: Performance and Stability Results
}

\author{
Lars Grüne $^{1}$, Simon Pirkelmann ${ }^{1}$ \\ ${ }^{1}$ Chair of Applied Mathematics, Mathematical Institute, University of Bayreuth, Germany
}

July 30, 2018

\begin{abstract}
We consider economic model predictive control (MPC) without terminal conditions for time-varying optimal control problems. Under appropriate conditions we prove that MPC yields initial pieces of approximately infinite horizon optimal trajectories, and that the optimal infinite horizon trajectory is practically asymptotically stable. The results are illustrated by two numerical examples, both motivated by energy efficient heating and cooling of a building.
\end{abstract}

\section{Contents}

1 Introduction

2 Problem statement

3 Economic model predictive control

4 MPC Performance estimates

5 MPC trajectory convergence

6 Examples

\section{Introduction}

In classical model predictive control (MPC) the aim is to track an a priori known reference trajectory of the system. The reference trajectory is either given by the control objective or prescribed by insight of the user into the process and can for example be a steady state at which the system should be stabilized. This steady state, in turn, may be computed via some form of optimization, e.g., as an optimal equilibrium of the system.

In contrast, in economic MPC the primary goal of the controller is not the stabilization of a pre-computed trajectory or steady state but instead the optimal performance of the controller with respect to an economic cost criterion, e.g. energy minimization or profit maximization. For time-invariant system dynamics this setting has been studied extensively and a number of performance results and conditions for convergence to an optimal steady state have been established both with [1] and without the use of terminal conditions [2].

It has been observed in various papers $[3,4,5,6]$ that even for time-invariant problems the optimal behaviour of the system may occur not at a steady state but rather at a time-varying periodic orbit that the system traverses cyclically. Consequently, it can no longer be expected that the system converges to a steady state but rather to the periodic orbit. In this paper, we go one step further and consider problems with time-varying data, in which the optimal behavior is a non-periodic time-varying trajectory.

In order to prove stability of model predictive controllers often terminal conditions are used in whose design explicit knowledge of the reference trajectory enters. Already for periodic optimal behavior this can be difficult, 
though for some problems it is in principle still doable as demonstrated in [7]. For general time-varying problems the computation of the optimal reference is in general an even more difficult task. For this reason the focus of research has turned to MPC schemes without terminal conditions.

In this paper we follow the same reasoning since we want to treat general time-varying systems that can exhibit complex optimal steering behavior. We do not assume a priori knowledge of the resulting trajectory of optimal operation — termed optimal trajectory in this paper — and thus entirely avoid the use of terminal conditions. Instead, we rely on the model predictive controller to find the optimal trajectory by itself, without providing it with any prior information about its location or structure. The questions to be addressed are under what conditions we can guarantee approximate optimal performance of the MPC controller and whether the trajectory generated by MPC converges to the optimal trajectory.

The paper is structured as follows. In the next section we introduce the mathematical formulation of the problem making use of an optimality notion that allows us to consider unbounded cost functionals on the infinite horizon and we define the optimal trajectory as a generalization of steady states or periodic orbits to the time-varying setting. Section 3 explains the MPC algorithm. In Section 4 we state performance estimates from a previous work [8] using two essential assumptions, the turnpike property and continuity of the optimal value functions. The next section establishes convergence of the economic MPC solution to the optimal trajectory which represents the main result of this paper. In the concluding section we give two examples that hint at a practical application from energy efficient building control.

\section{Problem statement}

We consider the discrete-time time-varying dynamics

$$
x(k+1)=f(k, x(k), u(k)), \quad x(0)=x,
$$

with $f: \mathbb{N}_{0} \times X \times U \rightarrow X$ and state space $X$ and control space $U$ are normed spaces. Here $k \in \mathbb{N}_{0}$ denotes time, $x(k) \in X$ the state of the system at time $k$ and $u(k) \in U$ the control.

A trajectory of the system starting at time $k$ from initial state $x \in X$ controlled by $u \in U^{N}, N \in \mathbb{N}$ is denoted by $x_{u}(\cdot ; k, x)$. We may omit the initial time and simply write $x_{u}(\cdot, x)$ when it is clear from the context. We want to incorporate state and control constraints in the problem statement. The sets of admissible states at time $k$ will be denoted by $\mathbb{X}(k) \subseteq X$ and the sets of admissible control values for $x \in \mathbb{X}(k)$ by $\mathbb{U}(k, x) \subseteq U$. By $\mathbb{U}^{N}(k, x)$ we denote the sets of admissible control sequences for initial state $x \in \mathbb{X}(k)$ up to time $k+N$, i.e. control sequences $u \in U^{N}$ satisfying

$$
u(j) \in \mathbb{U}\left(k+j, x_{u}(j ; k, x)\right) \text { and } x_{u}(j+1 ; k, x) \in \mathbb{X}(k+j+1)
$$

for all $j=0, \ldots, N-1$. The set $\mathbb{U}^{\infty}(k, x)$ denotes the natural extension of this definition to the infinite horizon.

Let $\ell: \mathbb{N}_{0} \times X \times U \rightarrow \mathbb{R}$ be the stage cost function and consider the cost functional

$$
J_{\infty}(k, x, u)=\sum_{j=0}^{\infty} \ell\left(k+j, x_{u}(j ; k, x), u(j)\right)
$$

The goal in our setting is to find a feasible control sequence $u \in \mathbb{U}^{\infty}(k, x)$ that minimizes $J_{\infty}(k, x, u)$. However, we first need to clarify what we mean by "minimizing" because for infinite optimal control sequences it is not obvious that $J_{\infty}(k, x, u)$ will attain a finite minimum at all. In fact with general stage cost the value of $J_{\infty}(k, x, u)$ may be infinite for all control sequences, so it is not directly possible to compare two control sequences based on their costs.

In the following we introduce a suitable notion of optimality that goes back to Gale[9] in the context of mathematical economics. The key idea is to consider not the total cost of two control sequences but instead to look at the difference of the costs. Although both control sequences in themselves generate infinite costs, the difference between the two can still be finite. A control sequence is considered to be optimal if its cost is overtaken by the cost of any other control sequence at some point.

Definition 1 (Overtaking optimality). Let $x \in \mathbb{X}(k)$ and consider a control sequence $u^{*} \in \mathbb{U}^{\infty}(k, x)$ with corresponding state trajectory $x_{u^{*}}(\cdot ; k, x)$. The pair $\left(x_{u^{*}}, u^{*}\right)$ is called overtaking optimal if

$$
\liminf _{K \rightarrow \infty} \sum_{j=0}^{K-1} \ell\left(k+j, x_{u}(j, x), u(j)\right)-\ell\left(k+j, x_{u^{*}}(j, x), u^{*}(j)\right) \geq 0
$$

for all $u \in \mathbb{U}^{\infty}(k, x)$. 
Definition 1 provides us with the ability to decide which of two infinite control sequences is better when starting at a fixed initial value $x$. The minimization in the following problem is to be understood in this overtaking optimal sense:

$$
\underset{u \in \mathbb{U}^{\infty}(k, x)}{\operatorname{minimize}} J_{\infty}(k, x, u)
$$

In the next definition the requirement of a fixed initial value is removed. This means we now look at all possible feasible trajectories that satisfy the dynamics of the system and pick from those the one that is optimal in the sense of Definition 1.

Definition 2 (Optimal operation). Let $x \in \mathbb{X}(k)$ and consider a control sequence $u^{*} \in \mathbb{U}^{\infty}(k, x)$ with corresponding state trajectory $x^{*}=x_{u^{*}}(\cdot ; k, x)$. We say the system (1) is optimally operated at $\left(x^{*}, u^{*}\right)$ if

$$
\liminf _{K \rightarrow \infty} \sum_{j=0}^{K-1} \ell\left(k+j, x_{u}\left(j, x^{\prime}\right), u(j)\right)-\ell\left(k+j, x^{*}(j), u^{*}(j)\right) \geq 0
$$

for all $x^{\prime} \in \mathbb{X}(k)$ and $u \in \mathbb{U}^{\infty}\left(k, x^{\prime}\right)$.

We will refer to the trajectory pair $\left(x^{*}, u^{*}\right)$ as the optimal trajectory. For the remainder of this paper we will assume that an optimal trajectory of the system always exists. Similarly, we assume a solution of problem (4) exists, which will be denoted by $u_{\infty}^{*}$.

The optimal trajectory can be considered a generalization of an optimal equilibrium or an optimal periodic orbit that may be present in the case of time-invariant systems, see e.g. [3]. In the classical time-invariant setting there may for example exist an optimal equilibrium at which the system can be operated at minimal cost for an infinite horizon. Then for any given initial condition we want to find a control sequence that brings the state to the optimal equilibrium.

In the same way in our setting an optimal trajectory exhibits the best performance in the long run. The question is how it connects to the solution of problem (4). In Section 4 we will introduce the turnpike property for infinite horizon. This key assumption guarantees that the solution of problem (4) converges to the optimal trajectory. This means we can reach the optimal operating behavior for a system by solving an infinite horizon optimal control problem. However, in general this is a very hard problem. In the following section we will introduce model predictive control as a method that aims to solve this problem by reduction to a finite horizon.

\section{Economic model predictive control}

In this paper we want to show that an approximate solution to problem (4) can be obtained by using model predictive control (MPC). The idea is introduced in the following: Instead of solving the problem on the infinite horizon we fix $N \in \mathbb{N}$ and consider the following cost functional.

Definition 3 (MPC cost functional). The MPC cost functional is defined as

$$
J_{N}(k, x, u)=\sum_{j=0}^{N-1} \ell\left(k+j, x_{u}(j ; k, x), u(j)\right)
$$

Then in each step of the MPC algorithm the following optimization problem is solved.

Definition 4 (MPC optimal control problem and optimal value function). Consider the problem

$$
\underset{u \in \mathbb{U}^{N}(k, x)}{\operatorname{minimize}_{N}} J_{N}(k, x, u)
$$

The corresponding optimal value function is defined by

$$
V_{N}(k, x):=\inf _{u \in \mathbb{U}^{N}(k, x)} J_{N}(k, x, u) .
$$

We will assume that the minimizer of this problem always exists and denote it by $u_{N}^{*}$, or else $u_{N, x}^{*}$ in case we want to stress the dependence on the the initial state $x$. Note that the optimal control satisfies $V_{N}(k, x)=J_{N}\left(k, x, u_{N, x}^{*}\right)$.

We remark that here we use the classical notion of optimality again since for finite horizon the cost functional cannot attain an infinite value. As a general rule we will need overtaking optimality only when optimizing over an infinite horizon, otherwise the usual notion of optimality suffices. 
(4).

The model predictive control algorithm given in Algorithm ?? produces an approximate solution to problem

The trajectory of the system generated in this way is called closed loop. We will denote it by $x_{\mu_{N}}(\cdot, x)$ for initial value $x=x\left(k_{0}\right) \in \mathbb{X}\left(k_{0}\right)$. The cost of the closed loop for $L$ time steps is defined by

$$
J_{L}^{c l}\left(k_{0}, x, \mu_{N}\right)=\sum_{j=0}^{L-1} \ell\left(k_{0}+j, x_{\mu_{N}}(j, x), \mu_{N}\left(k_{0}+j, x_{\mu_{N}}(j, x)\right)\right) .
$$

The aim of this paper is to study how good the approximation generated by the MPC algorithm is compared to the exact solution of the infinite horizon problem. In particular we want to address the following questions:

- How does the cost of the MPC closed loop compare to the cost of the solution on infinite horizon? This question is of special interest for problems in an economic setting. Here the behavior of the state trajectories is of secondary importance, instead we are more interested in finding a solution that generates the lowest possible costs. This question will be dealt with in Section 4.

- Does the closed loop converge to the optimal trajectory?

We also want to find out under what conditions we can establish convergence not only of the cost but also of the state. This is of particular interest since the optimal trajectory does not appear in the MPC problem from Definition 4. Instead, the algorithm finds the optimal trajectory by itself. In this respect, economic MPC differs from classical stabilizing MPC approaches, which require a priori knowledge about the optimal trajectory. An answer to this question will be given in Section 5 .

\section{MPC Performance estimates}

In this section we will deal with the question whether the cost of the MPC closed loop approximates the cost of the optimal trajectory on the infinite horizon. The key idea to bring together the trajectories of the MPC problem and the problem on an infinite horizon is to look at the turnpike property. It states that optimal trajectories converge on both finite and infinite horizon against the optimal trajectory $\left(x^{*}, u^{*}\right)$. An illustration of this can be found in Figure 1. The bound on the distance to the optimal trajectory is written in terms of a comparison function as defined in the following.

Definition 5 (Comparison functions). We define the following classes functions: $\mathcal{K}:=\left\{\alpha: \mathbb{R}_{0}^{+} \rightarrow \mathbb{R}_{0}^{+} \mid \alpha\right.$ is continuous and strictly increasing with $\left.\alpha(0)=0\right\}$

$\mathcal{K}_{\infty}:=\left\{\alpha: \mathbb{R}_{0}^{+} \rightarrow \mathbb{R}_{0}^{+} \mid \alpha \in \mathcal{K}\right.$ unbounded $\}$

$\mathcal{L}:=\left\{\delta: \mathbb{R}_{0}^{+} \rightarrow \mathbb{R}_{0}^{+} \mid \delta\right.$ is continuous and strictly decreasing with $\left.\lim _{t \rightarrow \infty} \delta(t)=0\right\}$

$\mathcal{K} \mathcal{L}:=\left\{\beta: \mathbb{R}_{0}^{+} \times \mathbb{R}_{0}^{+} \rightarrow \mathbb{R}_{0}^{+} \mid \beta\right.$ is continuous, $\left.\beta(\cdot, t) \in \mathcal{K}, \beta(r, \cdot) \in \mathcal{L}\right\}$

For the distance between two pairs $\left(x_{1}, u_{1}\right),\left(x_{2}, u_{2}\right) \in X \times U$ we will use the notation

$$
\left|\left(x_{1}, u_{1}\right)\right|_{\left(x_{2}, u_{2}\right)}:=\left\|x_{1}-x_{2}\right\|+\left\|u_{1}-u_{2}\right\|
$$

and similarly for $x_{1}, x_{2} \in X$

$$
\left|x_{1}\right|_{x_{2}}:=\left\|x_{1}-x_{2}\right\|
$$

Definition 6 (Turnpike property). Consider a trajectory pair $\left(x^{*}, u^{*}\right)$ at which the system (1) is optimally operated. We say that an optimal control problem has the turnpike property at $\left(x^{*}, u^{*}\right)$ if the following hold: There exists $\sigma \in \mathcal{L}$ such that for each $k \in \mathbb{N}_{0}$, each optimal trajectory $x_{u_{N}^{*}}(\cdot, x), x \in \mathbb{X}(k)$ and all $N, P \in \mathbb{N}$ there is a set $\mathcal{Q}(k, x, P, N) \subseteq\{0, \ldots, N\}$ with $\# \mathcal{Q}(k, x, P, N) \leq P$ and

$$
\left|\left(x_{u_{N}^{*}}(M, x), u_{N}^{*}(M)\right)\right|_{\left(x^{*}(k+M), u^{*}(k+M)\right)} \leq \sigma(P)
$$

for all $M \in\{0, \ldots, N\} \backslash \mathcal{Q}(k, x, P, N)$.

The definition can be extended to the infinite horizon. Here an optimal control problem has the turnpike property, if there exists $\rho \in \mathcal{L}$ such that for each $k \in \mathbb{N}_{0}$, each optimal trajectory $x_{u_{\infty}^{*}}(\cdot, x), x \in \mathbb{X}(k)$ and all $P \in \mathbb{N}$ there is a set $\mathcal{Q}(k, x, P, \infty) \subseteq \mathbb{N}_{0}$ with $\# \mathcal{Q}(k, x, P, \infty) \leq P$ and

$$
\left|\left(x_{u_{\infty}^{*}}(M, x), u_{\infty}^{*}(M)\right)\right|_{\left(x^{*}(k+M), u^{*}(k+M)\right)} \leq \rho(P)
$$

for all $M \in \mathbb{N}_{0} \backslash \mathcal{Q}(k, x, P, \infty)$. 


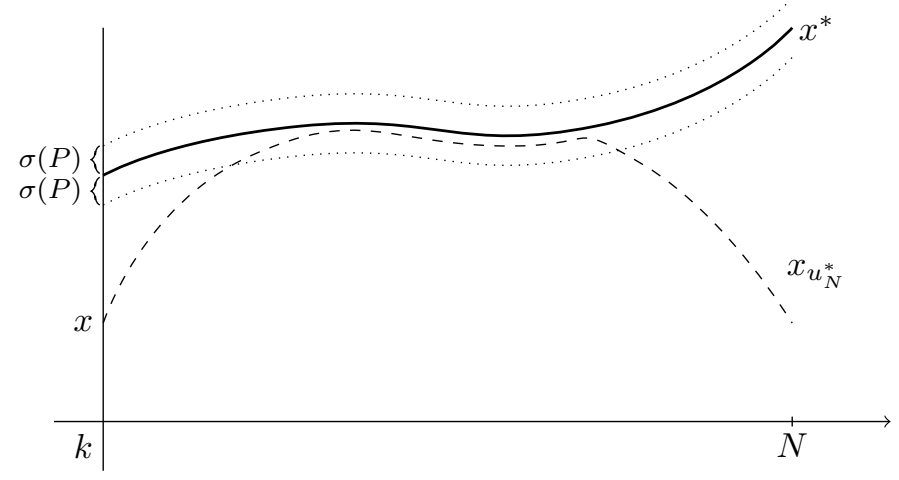

Figure 1: Illustration of the finite horizon turnpike property for time-varying systems.

Assumption 1 (Turnpike property for MPC problem). We assume the MPC problem from Definition 4 has the turnpike property.

Assumption 2 (Turnpike property for problem on infinite horizon). We assume the optimal control problem (4) has the turnpike property on infinite horizon.

While the turnpike property is a very convenient assumption it is at the same time not unreasonable. Turnpike properties of optimal control problems can be numerically observed for many practical problems. In addition, there are results that prove the existence of turnpike behavior for dissipative systems, see e.g. [10].

Note that the infinite horizon turnpike property can also be regarded as a convergence assumption of the solution of the infinite horizon problem (4) to the optimal trajectory. This is evident because the turnpike property requires that the distance between the optimal trajectory and the trajectory generated by $u_{\infty}^{*}$ can only be large for a finite number of points but this can only hold for a convergent trajectory.

The second ingredient for proving performance estimates for the MPC closed loop is continuity of the optimal value function. Before defining this continuity notion we first introduce a shifted stage cost function obtained by subtracting the cost of the optimal trajectory, as well as the corresponding cost functionals and optimal value function.

Definition 7 (Shifted cost). We define the shifted stage cost as

$$
\hat{\ell}(k, x(k), u(k)):=\ell(k, x(k), u(k))-\ell\left(k, x^{*}(k), u^{*}(k)\right)
$$

and the shifted cost functional as

$$
\hat{J}_{N}(k, x, u):=\sum_{j=0}^{N-1} \hat{\ell}\left(k+j, x_{u}(j ; k, x), u(j)\right) .
$$

The corresponding shifted optimal value function is given by

$$
\hat{V}_{N}(k, x):=\inf _{u \in \mathbb{U}^{N}(k, x)} \hat{J}_{N}(k, x, u) .
$$

In the same way for the infinite horizon we define

$$
\hat{J}_{\infty}(k, x, u):=\sum_{j=0}^{\infty} \hat{\ell}\left(k+j, x_{u}(j ; k, x), u(j)\right) .
$$

and

$$
\hat{V}_{\infty}(k, x):=\inf _{u \in \mathbb{U}^{\infty}(k, x)} \hat{J}_{\infty}(k, x, u) .
$$

We will formulate our results in terms of this shifted function. The reason for this change is that the optimal value function of the infinite horizon problem does not necessarily attain a finite value but it can be shown that under the turnpike assumption the shifted optimal value function $\hat{V}_{\infty}(k, x)$ is finite for all $x \in \mathbb{X}(k)$, see [8].

It should be noted that the solution of the shifted problem coincides with the solution of the MPC problem (7). This is important for practical considerations since it means we do not need to know the optimal trajectory $\left(x^{*}, u^{*}\right)$ in order to solve the problem. 
Assumption 3 (Continuity property of the optimal value function). We assume that the optimal value function $\hat{V}_{N}$ is (approximately) continuous at $x^{*}$ in the following uniform way: for each $k \in \mathbb{N}_{0}$ there is an open ball $\mathcal{B}_{\varepsilon}\left(x^{*}(k)\right), \varepsilon>0$, around $x^{*}(k)$ and a function $\gamma_{V}: \mathbb{R}_{0}^{+} \times \mathbb{R}_{0}^{+} \rightarrow \mathbb{R}_{0}^{+}$with $\gamma_{V}(N, r) \rightarrow 0$ if $N \rightarrow \infty$ and $r \rightarrow 0$, and $\gamma_{V}(\cdot, r), \gamma_{V}(N, \cdot)$ monotonous for fixed $r$ and $N$, such that for all $x \in \mathcal{B}_{\varepsilon}\left(x^{*}(k)\right) \cap \mathbb{X}(k)$ and all $N \in \mathbb{N}$ the inequality

$$
\left|\hat{V}_{N}(k, x)-\hat{V}_{N}\left(k, x^{*}(k)\right)\right| \leq \gamma_{V}\left(N,|x|_{x^{*}(k)}\right)
$$

holds.

Moreover, we also assume approximate continuity of the optimal value function on the infinite horizon: for each $k \in \mathbb{N}_{0}$ there is an open ball $\mathcal{B}_{\varepsilon}\left(x^{*}(k)\right), \varepsilon>0$, around $x^{*}(k)$ and a function $\omega_{V} \in \mathcal{K}_{\infty}$ such that for all $x \in \mathcal{B}_{\varepsilon}\left(x^{*}(k)\right) \cap \mathbb{X}(k)$ it holds

$$
\left|\hat{V}_{\infty}(k, x)-\hat{V}_{\infty}\left(k, x^{*}(k)\right)\right| \leq \omega_{V}\left(|x|_{x^{*}(k)}\right) .
$$

Using the turnpike and continuity properties one can prove the next result. Here we avoid duplicating the preliminary lemmas that lead to this results and also will not give the proofs. These can be found in [8].

Theorem 1 (see Theorem 1 in [8]). Let Assumptions 1 and 3 hold. Then for each $k \in \mathbb{N}_{0}$ and each sufficiently large $N \in \mathbb{N}$ the closed loop cost satisfies

$$
\hat{J}_{L}^{c l}\left(k, x, \mu_{N}\right) \leq \hat{V}_{\infty}(k, x)-\hat{V}_{\infty}\left(k+L, x_{\mu_{N}}(L, x)\right)+L \delta(N)
$$

with a function $\delta \in \mathcal{L}$.

As outlined in the discussion following the proof of the theorem in [8], this implies that the MPC closed loop trajectory approximates the cost of the infinite horizon optimal trajectory at least on finite horizons. More specifically, consider a control sequence that consists for the first $L$ steps of the MPC feedback solution and after that of the solution of the infinite horizon problem starting in $\tilde{x}=x_{\mu_{N}}(L, x)$ at time $k+L$ :

$$
\bar{u}(j):= \begin{cases}\mu_{N}\left(k+j, x_{\mu_{N}}(j, x)\right), & j=0, \ldots, L-1 \\ u_{\infty, \tilde{x}}^{*}(j), & j \geq L\end{cases}
$$

According to the theorem the cost of this control sequence is (up to the error term $L \delta(N)$ ) comparable to the cost of the optimal control sequence that solves the infinite horizon problem (4).

\section{MPC trajectory convergence}

In this section we show that not only the cost of the MPC trajectory approximates the cost of the infinite horizon optimal trajectory, but we can also establish convergence of the trajectory itself. This is formalized by using the notion of P-practical asymptotic stability and Lyapunov functions. For the definition of stability we substitute the feedback for the control in system (1), i.e. we consider the feedback controlled system

$$
x^{+}=f\left(k, x, \mu_{N}(k, x)\right)=: g(k, x) .
$$

The following definitions and the theorem are taken from [11].

Definition 8 (Forward invariance). We say a family of sets $Y(k) \subseteq X, k \in \mathbb{N}_{0}$ is forward invariant if $g(k, x) \in Y(k+1)$ for all $k \in \mathbb{N}_{0}$ and all $x \in Y(k)$.

Definition 9 (Uniform P-practical asymptotic stability). ${ }^{1}$ Let $Y(k)$ be a forward invariant family of sets and let $P(k) \subset Y(k)$ be subsets of $Y(k)$. Then we say that a trajectory $x^{*}$ with $x^{*}(k) \in Y(k)$ is $P$-practically uniformly asymptotically stable on $Y(k)$ if there exists $\beta \in \mathcal{K} \mathcal{L}$ such that

$$
\left|x\left(k ; k_{0}, x_{0}\right)\right|_{x^{*}(k)} \leq \beta\left(\left|x_{0}\right|_{x^{*}\left(k_{0}\right)}, k-k_{0}\right)
$$

holds for all $x_{0} \in Y\left(k_{0}\right)$ and all $k_{0}, k \in \mathbb{N}_{0}$ with $k \geq k_{0}$ and $x\left(k ; k_{0}, x_{0}\right) \notin P(k)$.

Uniform asymptotic stability is ensured by the existence of a Lyapunov function.

Definition 10 (Uniform time-varying Lyapunov function). ${ }^{2}$ Let subsets $S(k) \subseteq X$ and define $\mathcal{S}:=\{(k, x) \mid k \in$ $\left.\mathbb{N}_{0}, x \in S(k)\right\}$. A function $V: \mathcal{S} \rightarrow \mathbb{R}_{0}^{+}$is called uniform time-varying Lyapunov function on $S(k)$ if the following conditions are satisfied:

\footnotetext{
${ }^{1}$ cf. Definition 2.17 in [11]

${ }^{2}$ cf. Definition 2.21 in [11]
} 
1. There exist functions $\alpha_{1}, \alpha_{2} \in \mathcal{K}_{\infty}$ such that

$$
\alpha_{1}\left(|x|_{x^{*}(k)}\right) \leq V(k, x) \leq \alpha_{2}\left(|x|_{x^{*}(k)}\right)
$$

holds for all $k \in \mathbb{N}_{0}$ and all $x \in S(k)$.

2. There exists a function $\alpha_{V} \in \mathcal{K}$ such that

$$
V(k+1, g(k, x)) \leq V(k, x)-\alpha_{V}\left(|x|_{x^{*}(k)}\right)
$$

holds for all $k \in \mathbb{N}_{0}$ and all $x \in S(k)$ with $g(k, x) \in S(n+1)$.

Theorem 2 (P-practical asymptotic stability). ${ }^{3}$ Consider forward invariant families of sets $Y(k)$ and $P(k) \subset$ $Y(k), k \in \mathbb{N}_{0}$, and $x^{*}(k) \in P(k)$. If there exists a uniform time-varying Lyapunov function $V$ on $S(k)=$ $Y(k) \backslash P(k)$ then $x^{*}$ is P-practically asymptotically stable on $Y(k)$.

We will show that a modified optimal value function related to the optimal value function of the original MPC problem is a Lyapunov function for the feedback controlled system. An essential assumption we make is strict dissipativity of the system as introduced in the following.

Assumption 4 (Strict dissipativity). The system (1) is strictly dissipative with respect to the supply rate $s(k, x, u)=\hat{\ell}(k, x, u)$ and the optimal trajectory $\left(x^{*}, u^{*}\right)$, i.e. there exists a storage function $\lambda: \mathbb{N}_{0} \times X \rightarrow \mathbb{R}$ bounded from below on $X$ and $\alpha \in \mathcal{K}_{\infty}$ such that for all $k \in \mathbb{N}_{0}$ and all $(x, u) \in \mathbb{X}(k) \times \mathbb{U}(k, x)$ the following holds:

$$
\lambda(k+1, f(k, x, u))-\lambda(k, x) \leq s(k, x, u)-\alpha\left(|(x, u)|_{\left(x^{*}(k), u^{*}(k)\right)}\right)
$$

holds for all $k \in \mathbb{N}_{0}$ and all $(x, u) \in \mathbb{X}(k) \times \mathbb{U}(k, x)$.

Using the storage function $\lambda$ from the previous definition we introduce a modified MPC stage cost.

Definition 11 (Modified MPC cost functional). The modified stage cost $\tilde{\ell}$ is given by

$$
\tilde{\ell}(k, x, u)=\hat{\ell}(k, x, u)+\lambda(k, x)-\lambda(k+1, f(k, x, u)) .
$$

The modified MPC cost functional is defined as

$$
\tilde{J}_{N}(k, x, u)=\sum_{j=0}^{N-1} \tilde{\ell}\left(k+j, x_{u}(j ; k, x), u(j)\right) .
$$

Definition 12 (Modified MPC optimal control problem). We consider the modified MPC problem

$$
\min _{u \in \mathbb{U}^{N}(k, x)} \tilde{J}_{N}(k, x, u)
$$

and the corresponding modified optimal value function

$$
\tilde{V}_{N}(k, x):=\inf _{u \in \mathbb{U}^{N}(k, x)} \tilde{J}_{N}(k, x, u) .
$$

In the following we will state several assumptions for this modified problem in order to facilitate the proofs. In addition to the turnpike property from Assumption 1 we demand that the modified problem also has the turnpike property.

Assumption 5 (Turnpike property for the modified MPC problem). The modified optimal control problem from Definition 12 has the turnpike property. For the modified problem we will denote the set $\mathcal{Q}$ by $\tilde{\mathcal{Q}}$ and the bound $\sigma$ by $\tilde{\sigma}$.

Moreover, we make two assumptions for the modified stage cost and the modified optimal value function.

Assumption 6 (Modified cost bounded from above). We assume there exists $\alpha_{u} \in \mathcal{K}_{\infty}$ such that the modified stage cost satisfies

$$
\tilde{\ell}(k, x, u) \leq \alpha_{u}\left(|(x, u)|_{\left(x^{*}(k), u^{*}(k)\right)}\right)
$$

for all $k \in \mathbb{N}_{0}$ and all $(x, u) \in \mathbb{X}(k) \times \mathbb{U}(k, x)$.

\footnotetext{
${ }^{3}$ cf. Theorem 2.23 in [11]
} 
Assumption 7 (Continuity of $\tilde{V}_{N}$ at $x^{*}$ ). We assume there exists $\gamma_{\tilde{V}}$ such that for each $k \in \mathbb{N}_{0}, N \in \mathbb{N}$ and $x \in \mathbb{X}$ the following holds

$$
\left|\tilde{V}_{N}(k, x)-\tilde{V}_{N}\left(k, x^{*}(k)\right)\right| \leq \gamma_{\tilde{V}}\left(|x|_{x^{*}(k)}\right)
$$

Remark 1 (Modified cost along optimal trajectory). From Assumptions 4 and 6 it follows that the modified cost along the optimal trajectory pair $\left(x^{*}, u^{*}\right)$ satisfies

$$
\tilde{\ell}\left(k, x^{*}(k), u^{*}(k)\right)=0
$$

for all $k \in \mathbb{N}_{0}$. This also implies that

$$
\tilde{V}_{N}\left(k, x^{*}(k)\right)=0
$$

for all $k \in \mathbb{N}_{0}$ and for every $N \in \mathbb{N}$.

Remark 2 (Difference between different continuity assumptions). Note the difference between the two continuity assumptions from Assumption 3 and Assumption 7. The continuity assumption for the modified problem is independent of the horizon $N$.

The following preparatory lemma shows that the initial cost (up to some time instant $M$ ) of two optimal trajectories with different horizon length of the modified problem is nearly identical.

Lemma 1. Let Assumptions 5 and 7 hold. Then

$$
\tilde{J}_{M}\left(k, x, \tilde{u}_{N}^{*}\right)=\tilde{J}_{M}\left(k, x, \tilde{u}_{N+1}^{*}\right)+R_{5}(k, x, M, N)
$$

where the error term satisfies $\left|R_{5}(k, x, M, N)\right| \leq 2 \gamma_{\tilde{V}}(\tilde{\sigma}(P))$ for all $k \in \mathbb{N}_{0}$, all $N \in \mathbb{N}$, all $P \in \mathbb{N}$ sufficiently large, all $x \in \mathbb{X}(k)$ and all $M \in\{0, \ldots, N\} \backslash(\tilde{\mathcal{Q}}(k, x, P, N) \cup \tilde{\mathcal{Q}}(k, x, P, N+1))$.

Proof. Let $\tilde{u}_{N}^{*}$ and $\tilde{u}_{N+1}^{*}$ denote the optimal solutions of problem (12) with horizon $N$ and $N+1$, respectively. From the finite horizon dynamic programming principle we obtain that $u=\tilde{u}_{N}^{*}$ is a minimizer of $\tilde{J}_{M}(k, x, u)+$ $\tilde{V}_{N-M}\left(k+M, x_{u}(M, x)\right)$. In particular it holds that

$$
\tilde{J}_{M}\left(k, x, \tilde{u}_{N}^{*}\right)+\tilde{V}_{N-M}\left(k+M, x_{\tilde{u}_{N}^{*}}(M, x)\right) \leq \tilde{J}_{M}\left(k, x, \tilde{u}_{N+1}^{*}\right)+\tilde{V}_{N-M}\left(k+M, x_{\tilde{u}_{N+1}^{*}}(M, x)\right) .
$$

Now consider

$$
R_{1}(k, x, M, N):=\tilde{V}_{N-M}\left(k+M, x_{\tilde{u}_{N}^{*}}(M, x)\right)-\tilde{V}_{N-M}\left(k+M, x^{*}(k+M)\right)
$$

and

$$
R_{2}(k, x, M, N):=\tilde{V}_{N-M}\left(k+M, x_{\tilde{u}_{N+1}^{*}}(M, x)\right)-\tilde{V}_{N-M}\left(k+M, x^{*}(k+M)\right) .
$$

Inserting the definition of $R_{1}$ and $R_{2}$ into (21) we obtain

$$
\begin{aligned}
& \tilde{J}_{M}\left(k, x, \tilde{u}_{N}^{*}\right)+\tilde{V}_{N-M}\left(k+M, x^{*}(k+M)\right)+R_{1}(k, x, M, N) \\
& \leq \tilde{J}_{M}\left(k, x, \tilde{u}_{N+1}^{*}\right)+\tilde{V}_{N-M}\left(k+M, x^{*}(k+M)\right)+R_{2}(k, x, M, N)
\end{aligned}
$$

which is equivalent to

$$
\tilde{J}_{M}\left(k, x, \tilde{u}_{N}^{*}\right) \leq \tilde{J}_{M}\left(k, x, \tilde{u}_{N+1}^{*}\right)-R_{1}(k, x, M, N)+R_{2}(k, x, M, N) .
$$

The above equations are true for every $M \in\{0, \ldots, N\}$. For $M \in\{0, \ldots, N\} \backslash(\tilde{\mathcal{Q}}(k, x, P, N) \cup \tilde{\mathcal{Q}}(k, x, P, N+1))$ we know from Assumption 5 that $\left|\left(x_{\tilde{u}_{N}^{*}}(M, x), \tilde{u}_{N}^{*}(M)\right)\right|_{\left(x^{*}(k+M), u^{*}(k+M)\right)} \leq \tilde{\sigma}(P)$, and in particular $\left|x_{\tilde{u}_{N}^{*}}(M, x)\right|_{x^{*}(k+M)} \leq$ $\tilde{\sigma}(P)$, i.e. we have a bound on the distance of $x_{\tilde{u}_{N}^{*}}(M, x)$ to the optimal trajectory $x^{*}$. Using Assumption 7 we obtain

$$
\left|R_{1}(k, x, M, N)\right| \leq \gamma_{\tilde{V}}(\tilde{\sigma}(P)) .
$$

The same holds when considering the optimal trajectory $\tilde{u}_{N+1}^{*}$ yielding the estimate

$$
\left|R_{2}(k, x, M, N)\right| \leq \gamma_{\tilde{V}}(\tilde{\sigma}(P))
$$

For the converse inequality we use the dynamic programming principle once more together with the fact that $u=\tilde{u}_{N+1}^{*}$ minimizes the expression $\tilde{J}_{M}(k, x, u)+\tilde{V}_{N+1-M}\left(k+M, x_{u}(M, x)\right)$ which implies that

$$
\tilde{J}_{M}\left(k, x, \tilde{u}_{N+1}^{*}\right)+\tilde{V}_{N+1-M}\left(k+M, x_{\tilde{u}_{N+1}^{*}}(M, x)\right) \leq \tilde{J}_{M}\left(k, x, \tilde{u}_{N}^{*}\right)+\tilde{V}_{N+1-M}\left(k+M, x_{\tilde{u}_{N}^{*}}(M, x)\right) .
$$

Defining

$$
R_{3}(k, x, M, N):=\tilde{V}_{N+1-M}\left(k+M, x_{\tilde{u}_{N+1}^{*}}(M, x)\right)-\tilde{V}_{N+1-M}\left(k+M, x^{*}(k+M)\right)
$$


and

$$
R_{4}(k, x, M, N):=\tilde{V}_{N+1-M}\left(k+M, x_{\tilde{u}_{N}^{*}}(M, x)\right)-\tilde{V}_{N+1-M}\left(k+M, x^{*}(k+M)\right)
$$

we can estimate

$$
\begin{aligned}
& \tilde{J}_{M}\left(k, x, \tilde{u}_{N+1}^{*}\right)+\tilde{V}_{N+1-M}\left(k+M, x^{*}(k+M)\right)+R_{3}(k, x, M, N) \\
& \leq \tilde{J}_{M}\left(k, x, \tilde{u}_{N}^{*}\right)+\tilde{V}_{N+1-M}\left(k+M, x^{*}(k+M)\right)+R_{4}(k, x, M, N) \\
\Leftrightarrow & \tilde{J}_{M}\left(k, x, \tilde{u}_{N+1}^{*}\right) \leq \tilde{J}_{M}\left(k, x, \tilde{u}_{N}^{*}\right)-R_{3}(k, x, M, N)+R_{4}(k, x, M, N) .
\end{aligned}
$$

Analogously to the above discussion we obtain the bounds

$$
\left|R_{3}(k, x, M, N)\right| \leq \gamma_{\tilde{V}}(\tilde{\sigma}(P))
$$

and

$$
\left|R_{4}(k, x, M, N)\right| \leq \gamma_{\tilde{V}}(\tilde{\sigma}(P))
$$

for every $M \in\{0, \ldots, N\} \backslash(\tilde{\mathcal{Q}}(k, x, P, N) \cup \tilde{\mathcal{Q}}(k, x, P, N+1))$. Finally, combining the inequalities (22) and (23) leads to

$$
\begin{aligned}
& \left|R_{5}(k, x, M, N)\right|=\left|\tilde{J}_{M}\left(k, x, \tilde{u}_{N}^{*}\right)-\tilde{J}_{M}\left(k, x, \tilde{u}_{N+1}^{*}\right)\right| \\
& \leq \max \left\{\left|-R_{1}(k, x, M, N)+R_{2}(k, x, M, N)\right|,\left|-R_{3}(k, x, M, N)+R_{4}(k, x, M, N)\right|\right\} \\
& \leq \max \left\{\left|R_{1}(k, x, M, N)\right|+\left|R_{2}(k, x, M, N)\right|,\left|R_{3}(k, x, M, N)\right|+\left|R_{4}(k, x, M, N)\right|\right\} \\
& \leq \max \left\{2 \gamma_{\tilde{V}}(\tilde{\sigma}(P)), 2 \gamma_{\tilde{V}}(\tilde{\sigma}(P))\right\} \\
& =2 \gamma_{\tilde{V}}(\tilde{\sigma}(P)) .
\end{aligned}
$$

This concludes the proof.

Using this result we can prove the following lemma, which states that the optimal value functions for the modified problem yields almost the same value for different horizons $N$ and $N+1$.

Lemma 2. Let Assumption 6 and those of Lemma 1 hold. Then the equation

$$
\tilde{V}_{N+1}(k, x)=\tilde{V}_{N}(k, x)+R_{6}(k, x, M, N)
$$

holds with $\left|R_{6}(k, x, M, N)\right| \leq 4 \gamma_{\tilde{V}}(\tilde{\sigma}(P))$ for all $k \in \mathbb{N}_{0}$, all $N \in \mathbb{N}$, all $P \in \mathbb{N}$ sufficiently large, all $x \in \mathbb{X}(k)$ and all $M \in\{0, \ldots, N\} \backslash(\tilde{\mathcal{Q}}(k, x, P, N) \cup \tilde{\mathcal{Q}}(k, x, P, N+1))$.

Proof. Let $k \in \mathbb{N}_{0}$ and let $x \in \mathbb{X}(k)$. We first consider the optimal value function with horizon length $N$. From the dynamic programming principle it follows for every $M \in\{0, \ldots, N\}$ that

$$
\tilde{V}_{N}(k, x)=\tilde{J}_{M}\left(k, x, \tilde{u}_{N}^{*}\right)+\tilde{V}_{N-M}\left(k+M, x_{\tilde{u}_{N}^{*}}(M, x)\right) .
$$

We define

$$
R_{1}(k, x, M, N):=\tilde{V}_{N-M}\left(k+M, x_{\tilde{u}_{N}^{*}}(M, x)\right)-\tilde{V}_{N-M}\left(k+M, x^{*}(k+M)\right)
$$

which can be bounded by

$$
\left|R_{1}(k, x, M, N)\right| \leq \gamma_{\tilde{V}}(\tilde{\sigma}(P))
$$

for $M \in\{0, \ldots, N\} \backslash \tilde{\mathcal{Q}}(k, x, P, N)$ as seen in the proof of Lemma 1.

Using the definition of $R_{1}$ we rewrite (24) to

$$
\begin{aligned}
\tilde{V}_{N}(k, x) & =\tilde{J}_{M}\left(k, x, \tilde{u}_{N}^{*}\right)+\tilde{V}_{N-M}\left(k+M, x^{*}(k+M)\right)+R_{1}(k, x, M, N) \\
& =\tilde{J}_{M}\left(k, x, \tilde{u}_{N}^{*}\right)+R_{1}(k, x, M, N)
\end{aligned}
$$

where we used Remark 1 in the last equality.

Now consider the optimal value function for horizon length $N+1$. Again, we apply the dynamic programming principle which yields

$$
\tilde{V}_{N+1}(k, x)=\tilde{J}_{M}\left(k, x, \tilde{u}_{N+1}^{*}\right)+\tilde{V}_{N+1-M}\left(k+M, x_{\tilde{u}_{N+1}^{*}}(M, x)\right)
$$

for every $M \in\{0, \ldots, N+1\}$. We define

$$
R_{3}(k, x, M, N):=\tilde{V}_{N+1-M}\left(k+M, x_{\tilde{u}_{N+1}^{*}}(M, x)\right)-\tilde{V}_{N+1-M}\left(k+M, x^{*}(k+M)\right)
$$


with the bound

$$
\left|R_{3}(k, x, M, N)\right| \leq \gamma_{\tilde{V}}(\tilde{\sigma}(P))
$$

for $M \in\{0, \ldots, N\} \backslash \tilde{\mathcal{Q}}(k, x, P, N+1)$ (cf. Lemma 1$)$.

Inserting the definition of $R_{3}$ into (26) and using Remark 1 we obtain

$$
\begin{aligned}
\tilde{V}_{N+1}(k, x) & =\tilde{J}_{M}\left(k, x, \tilde{u}_{N+1}^{*}\right)+\tilde{V}_{N+1-M}\left(k+M, x^{*}(k+M)\right)+R_{3}(k, x, M, N) \\
& =\tilde{J}_{M}\left(k, x, \tilde{u}_{N+1}^{*}\right)+R_{3}(k, x, M, N) .
\end{aligned}
$$

For $M \in\{0, \ldots, N\} \backslash(\tilde{\mathcal{Q}}(k, x, P, N) \cup \tilde{\mathcal{Q}}(k, x, P, N+1))$ we apply Lemma 1 to get

$$
\begin{aligned}
\tilde{V}_{N+1}(k, x) & =\tilde{J}_{M}\left(k, x, \tilde{u}_{N+1}^{*}\right)+R_{3}(k, x, M, N) \\
& =\tilde{J}_{M}\left(k, x, \tilde{u}_{N}^{*}\right)+R_{3}(k, x, M, N)-R_{5}(k, x, M, N) \\
& =\tilde{V}_{N}(k, x)-R_{1}(k, x, M, N)+R_{3}(k, x, M, N)-R_{5}(k, x, M, N)
\end{aligned}
$$

where the last equation follows with equation (25).

Finally, we define

$$
R_{6}(k, x, M, N):=-R_{1}(k, x, M, N)+R_{3}(k, x, M, N)-R_{5}(k, x, M, N)
$$

and from the bounds on $R_{1}, R_{3}$ and $R_{5}$ we get the bound

$$
\begin{aligned}
\left|R_{6}(k, x, M, N)\right| & =\left|-R_{1}(k, x, M, N)+R_{3}(k, x, M, N)-R_{5}(k, x, M, N)\right| \\
& \leq\left|R_{1}(k, x, M, N)\right|+\left|R_{3}(k, x, M, N)\right|+\left|R_{5}(k, x, M, N)\right| \\
& \leq \gamma_{\tilde{V}}(\tilde{\sigma}(P))+\gamma_{\tilde{V}}(\tilde{\sigma}(P))+2 \gamma_{\tilde{V}}(\tilde{\sigma}(P)) \\
& =4 \gamma_{\tilde{V}}(\tilde{\sigma}(P)) .
\end{aligned}
$$

This shows the assertion.

Remark 3. In the next lemma we will use both the turnpike property for the modified and the unmodified MPC problem. Note however, that Assumption 1 and Assumption 5 express two different turnpike properties with different bounds $\sigma$ and $\tilde{\sigma}$ and associated sets $\mathcal{Q}$ and $\tilde{\mathcal{Q}}$. For the proof of the following lemma we will need a common bound and a single set for both problems. This can be achieved by defining

$$
\bar{\sigma}:=\max \{\sigma, \tilde{\sigma}\}
$$

and

$$
\overline{\mathcal{Q}}(k, x, P, N):=\mathcal{Q}(k, x, P, N) \cup \tilde{\mathcal{Q}}(k, x, P, N) .
$$

Then the optimal trajectories of both problems from Definitions 4 and 12 satisfy

$$
\left|\left(x_{u_{N, x}^{*}}(M, x), u_{N, x}^{*}(M)\right)\right|_{\left(x^{*}(k+M), u^{*}(k+M)\right)} \leq \bar{\sigma}(P)
$$

and

$$
\mid\left(\left.x_{\tilde{u}_{N, x}^{*}}\left(M, x, \tilde{u}_{N, x}^{*}(M)\right)\right|_{\left(x^{*}(k+M), u^{*}(k+M)\right)} \leq \bar{\sigma}(P)\right.
$$

for all $M \in\{0, \ldots, N\} \backslash \overline{\mathcal{Q}}(k, x, P, N)$ and $\# \overline{\mathcal{Q}}(k, x, P, N) \leq 2 P$.

The next theorem shows that the initial piece of an optimal control trajectory which ends in a neighborhood of the optimal trajectory of the unmodified MPC problem yields approximately lower cost than all other trajectories ending in that neighborhood.

Theorem 3 (Initial piece of optimal trajectory ending near turnpike is optimal). Let $u_{N, x}^{*}$ denote the optimal trajectory of problem (4) and let Assumptions 1, 3 and 5 hold. Then for all $k \in \mathbb{N}_{0}$, all $x \in \mathbb{X}(k)$, all $N \in \mathbb{N}$, all $P \in \mathbb{N}$, all $M \in\{0, \ldots, N\} \backslash \overline{\mathcal{Q}}(k, x, P, N)$ and all $u \in \mathbb{U}^{M}(k, x)$ with $\left|x_{u}(M, x)\right|_{x^{*}(k+M)} \leq \bar{\sigma}(P)$ the estimate

$$
\hat{J}_{M}\left(k, x, u_{N, x}^{*}\right) \leq \hat{J}_{M}(k, x, u)+R_{7}(k, x, M, N)
$$

holds with $\left|R_{7}(k, x, M, N)\right| \leq 2 \gamma_{V}(N-M, \bar{\sigma}(P))$.

Proof. We prove the theorem by contradiction. Let $u_{N, x}^{*}$ denote the optimal solution of problem (4) and let $\bar{x}_{u^{*}}:=x_{u_{N, x}^{*}}(M, x)$ for $M \in\{0, \ldots, N\} \backslash \overline{\mathcal{Q}}(k, x, P, N)$. Then from Remark 3 we know that $\left|\bar{x}_{u^{*}}\right|_{x^{*}(k+M)} \leq \bar{\sigma}(P)$.

Now assume there exists a control sequence $u \in \mathbb{U}^{M}(k, x)$ with $\bar{x}_{u}:=x_{u}(M, x)$ satisfying $\left|\bar{x}_{u}\right|_{x^{*}(k+M)} \leq \bar{\sigma}(P)$ and

$$
\hat{J}_{M}(k, x, u)+R_{1}(k, x, M, N)+R_{2}(k, x, M, N)<\hat{J}_{M}\left(k, x, u_{N, x}^{*}\right) .
$$


with

$$
R_{1}(k, x, M, N):=\hat{V}_{N-M}\left(k+M, \bar{x}_{u}\right)-\hat{V}_{N-M}\left(k+M, x^{*}(k+M)\right)
$$

and

$$
R_{2}(k, x, M, N):=\hat{V}_{N-M}\left(k+M, x^{*}(k+M)\right)-\hat{V}_{N-M}\left(k+M, \bar{x}_{u^{*}}\right) .
$$

Using Assumption $3 R_{1}$ and $R_{2}$ can be bounded by

$$
\begin{aligned}
& \left|R_{1}(k, x, M, N)\right| \leq \gamma_{V}(N-M, \bar{\sigma}(P)) \\
& \left|R_{2}(k, x, M, N)\right| \leq \gamma_{V}(N-M, \bar{\sigma}(P)) .
\end{aligned}
$$

Consider

$$
\begin{aligned}
\hat{J}_{M}(k, x, u)+\hat{V}_{N-M}\left(k+M, \bar{x}_{u}\right)= & \hat{J}_{M}(k, x, u)+\hat{V}_{N-M}\left(k+M, x^{*}(k+M)\right)+R_{1}(k, x, M, N) \\
= & \hat{J}_{M}(k, x, u)+\hat{V}_{N-M}\left(k+M, \bar{x}_{u^{*}}\right) \\
& +R_{1}(k, x, M, N)+R_{2}(k, x, M, N) \\
& \stackrel{(28)}{<} \hat{J}_{M}\left(k, x, u_{N, x}^{*}\right)+\hat{V}_{N-M}\left(k+M, \bar{x}_{u^{*}}\right) \\
= & \hat{V}_{N}(k, x)
\end{aligned}
$$

where we used the dynamic programming principle for the last equation. But this contradicts the optimality of $u_{N, x}^{*}$ and thus the inequality

$$
\hat{J}_{M}\left(k, x, u_{N, x}^{*}\right) \leq \hat{J}_{M}(k, x, u)+R_{1}(k, x, M, N)+R_{2}(k, x, M, N)
$$

follows. Finally, define

$$
R_{7}(k, x, M, N):=R_{1}(k, x, M, N)+R_{2}(k, x, M, N)
$$

which can be bounded by

$$
\left|R_{7}(k, x, M, N)\right| \leq\left|R_{1}(k, x, M, N)\right|+\left|R_{2}(k, x, M, N)\right| \stackrel{(29)}{\leq} 2 \gamma_{V}(N-M, \bar{\sigma}(P)) .
$$

This concludes the proof.

So far we did not impose any assumptions on the storage function $\lambda$ from the strict dissipativity of the system. For the next lemma we will need that this function is continuous at the optimal trajectory.

Assumption 8 (Continuity of storage function $\lambda$ at $x^{*}$ ). Assume that the storage function $\lambda$ is continuous in the following sense: There exists $\gamma_{\lambda} \in \mathcal{K}_{\infty}$ such that for all $k \in \mathbb{N}$ and all $x \in \mathbb{X}$ it holds that

$$
\left|\lambda(k, x)-\lambda\left(k, x^{*}(k)\right)\right| \leq \gamma_{\lambda}\left(|x|_{x^{*}(k)}\right) .
$$

In our final preparatory lemma we consider a control sequence $\hat{u}$ that for the first part consists of the optimal control sequence $u_{N, x}^{*}$ of the unmodified problem until it is close to the optimal trajectory $x^{*}$. Then we control from the final point using the optimal control sequence of the modified problem. The lemma states that the resulting composite control sequence has almost the same cost as if we had controlled using the optimal control sequence of the modified problem for the whole horizon.

Lemma 3. Let Assumptions 1, 3, 5 and 8 hold and let $u_{N, x}^{*}$ and $\tilde{u}_{N, x}^{*}$ denote the optimal control sequences corresponding to problems (4) and (12). Let $N, P \in \mathbb{N}$ be arbitrary and for $M \in\{0, \ldots, N\} \backslash \overline{\mathcal{Q}}(k, x, P, N)$ define $\bar{x}_{u^{*}}:=x_{u_{N, x}^{*}}(M, x)$ and denote by $\bar{u}$ solution of the optimal control problem

$$
\min _{u \in \mathbb{U}^{N-M}\left(k+M, \bar{x}_{u^{*}}\right)} \tilde{J}_{N-M}\left(k+M, \bar{x}_{u^{*}}, u\right) .
$$

Then the composite control sequence $\hat{u} \in \mathbb{U}^{N}(k, x)$ defined by $\hat{u}(k)=u_{N, x}^{*}(k)$ for $k=\{0, \ldots, M-1\}$ and $\hat{u}(k+M)=\bar{u}(k)$ for $k=\{0, \ldots, N-M\}$ satisfies

$$
\tilde{J}_{N}(k, x, \hat{u})=\tilde{V}_{N}(k, x)+R_{8}(k, x, M, N)
$$

with

$$
\left|R_{8}(k, x, M, N)\right| \leq \gamma_{\tilde{V}}(\sigma(P))+\gamma_{\lambda}(\sigma(P))+\gamma_{\tilde{V}}(\tilde{\sigma}(P))+\gamma_{\lambda}(\tilde{\sigma}(P))+2 \gamma_{V}(N-M, \bar{\sigma}(P))
$$

for all $k \in \mathbb{N}_{0}$ and for all $x \in \mathbb{X}(k)$. 
Proof. We first prove " $\tilde{J}_{N}(k, x, \hat{u})+\varepsilon(N) \leq \tilde{V}_{N}(k, x)$ ": Using the definition of $\hat{u}$, noting that $\bar{u}$ is an optimal solution and inserting the definition of $\tilde{J}_{M}$ we obtain

$$
\begin{aligned}
\tilde{J}_{N}(k, x, \hat{u}) & =\tilde{J}_{M}\left(k, x, u_{N, x}^{*}\right)+\tilde{J}_{N-M}\left(k+M, \bar{x}_{u^{*}}, \bar{u}\right) \\
& =\tilde{J}_{M}\left(k, x, u_{N, x}^{*}\right)+\tilde{V}_{N-M}\left(k+M, \bar{x}_{u^{*}}\right) \\
& =\hat{J}_{M}\left(k, x, u_{N, x}^{*}\right)+\lambda(k, x)-\lambda\left(k+M, \bar{x}_{u^{*}}\right)+\tilde{V}_{N-M}\left(k+M, \bar{x}_{u^{*}}\right)
\end{aligned}
$$

Define

$$
R_{1}(k, x, M, N):=\tilde{V}_{N-M}\left(k+M, \bar{x}_{u^{*}}\right)-\tilde{V}_{N-M}\left(k+M, x^{*}(k+M)\right)
$$

and

$$
R_{2}(k, x, M, N):=\lambda\left(k+M, x^{*}(k+M)\right)-\lambda\left(k+M, \bar{x}_{u^{*}}\right) .
$$

Because of Assumption 1 we know the bound $\left|\bar{x}_{u^{*}}\right|_{x^{*}(k+M)} \leq \sigma(P)$ for $M \in\{0, \ldots, N\} \backslash \mathcal{Q}(k, x, P, N)$. Thus we can use the continuity of $\tilde{V}_{N-M}$ from Assumption 7 and the continuity of $\lambda$ from Assumption 8 to obtain the bounds

$$
\left|R_{1}(k, x, M, N)\right| \leq \gamma_{\tilde{V}}(\sigma(P))
$$

and

$$
\left|R_{2}(k, x, M, N)\right| \leq \gamma_{\lambda}(\sigma(P))
$$

Inserting $R_{1}$ and $R_{2}$ into (32) leads to

$$
\begin{aligned}
& \hat{J}_{M}\left(k, x, u_{N, x}^{*}\right)+\lambda(k, x)-\lambda\left(k+M, \bar{x}_{u^{*}}\right)+\tilde{V}_{N-M}\left(k+M, \bar{x}_{u^{*}}\right) \\
& =\hat{J}_{M}\left(k, x, u_{N, x}^{*}\right)+\lambda(k, x)-\lambda\left(k+M, x^{*}(k+M)\right)+\tilde{V}_{N-M}\left(k+M, x^{*}(k+M)\right) \\
& \quad+R_{1}(k, x, M, N)+R_{2}(k, x, M, N)
\end{aligned}
$$

Now consider the optimal solution $\tilde{u}_{N, x}^{*}$ of problem $(12)$, denote $\tilde{x}:=x_{\tilde{u}_{N, x}^{*}}(M, x)$ and define

$$
R_{3}(k, x, M, N):=\tilde{V}_{N-M}\left(k+M, x^{*}(k+M)\right)-\tilde{V}_{N-M}(k+M, \tilde{x})
$$

and

$$
R_{4}(k, x, M, N):=\lambda(k+M, \tilde{x})-\lambda\left(k+M, x^{*}(k+M)\right) .
$$

For $M \in\{0, \ldots, N\} \backslash \tilde{\mathcal{Q}}(k, x, P, N)$ we have the bound $|\tilde{x}| \leq \tilde{\sigma}(P)$ from Assumption 5 . Using again the continuity of $\tilde{V}_{N-M}$ and $\lambda$ from Assumptions 7 and 8 we can bound $R_{3}$ and $R_{4}$ by

$$
\left|R_{3}(k, x, M, N)\right| \leq \gamma_{\tilde{V}}(\tilde{\sigma}(P))
$$

and

$$
\left|R_{4}(k, x, M, N)\right| \leq \gamma_{\lambda}(\tilde{\sigma}(P)) .
$$

Continuing from (33) by inserting $R_{3}$ and $R_{4}$ yields

$$
\begin{aligned}
& \hat{J}_{M}\left(k, x, u_{N, x}^{*}\right)+\lambda(k, x)-\lambda\left(k+M, x^{*}(k+M)\right)+\tilde{V}_{N-M}\left(k+M, x^{*}(k+M)\right) \\
& \quad+R_{1}(k, x, M, N)+R_{2}(k, x, M, N) \\
& =\hat{J}_{M}\left(k, x, u_{N, x}^{*}\right)+\lambda(k, x)-\lambda(k+M, \tilde{x})+\tilde{V}_{N-M}(k+M, \tilde{x}) \\
& \quad+R_{1}(k, x, M, N)+R_{2}(k, x, M, N)+R_{3}(k, x, M, N)+R_{4}(k, x, M, N)
\end{aligned}
$$

Finally, using Theorem 3 for the control sequence $u=\tilde{u}_{N, x}^{*}$ we obtain for $M \in\{0, \ldots, N\} \backslash \overline{\mathcal{Q}}(k, x, P, N)$

$$
\begin{aligned}
& \hat{J}_{M}\left(k, x, u_{N, x}^{*}\right)+\lambda(k, x)-\lambda(k+M, \tilde{x})+\tilde{V}_{N-M}(k+M, \tilde{x}) \\
& \quad+R_{1}(k, x, M, N)+R_{2}(k, x, M, N)+R_{3}(k, x, M, N)+R_{4}(k, x, M, N) \\
& \leq \hat{J}_{M}\left(k, x, \tilde{u}_{N, x}^{*}\right)+\lambda(k, x)-\lambda(k+M, \tilde{x})+\tilde{V}_{N-M}(k+M, \tilde{x}) \\
& \quad+\underbrace{R_{1}(k, x, M, N)+R_{2}(k, x, M, N)+R_{3}(k, x, M, N)+R_{4}(k, x, M, N)+R_{7}(k, x, M, N)}_{=: \tilde{R}_{8}(k, x, M, N)} \\
& =\tilde{J}_{M}\left(k, x, \tilde{u}_{N, x}^{*}\right)+\tilde{V}_{N-M}(k+M, \tilde{x})+\tilde{R}_{8}(k, x, M, N) \\
& =\tilde{V}_{N}(k, x)+\tilde{R}_{8}(k, x, M, N)
\end{aligned}
$$

In summary, we have shown that

$$
\tilde{J}_{N}(k, x, \hat{u}) \leq \tilde{V}_{N}(k, x)+\tilde{R}_{8}(k, x, M, N)
$$


with

$$
\begin{aligned}
\left|\tilde{R}_{8}(k, x, M, N)\right| \leq & \left|R_{1}(k, x, M, N)\right|+\left|R_{2}(k, x, M, N)\right|+\left|R_{3}(k, x, M, N)\right|+\left|R_{4}(k, x, M, N)\right| \\
& +\left|R_{7}(k, x, M, N)\right| \\
\leq & \gamma_{\tilde{V}}(\sigma(P))+\gamma_{\lambda}(\sigma(P))+\gamma_{\tilde{V}}(\tilde{\sigma}(P))+\gamma_{\lambda}(\tilde{\sigma}(P))+2 \gamma_{V}(N-M, \bar{\sigma}(P))
\end{aligned}
$$

which holds for all $M \in\{0, \ldots, N\} \backslash \overline{\mathcal{Q}}(k, x, P, N)$. On the other hand from the definition of the optimal value function we know that

$$
\tilde{V}_{N}(k, x) \leq \tilde{J}_{N}(k, x, u)
$$

for all $u \in \mathbb{U}^{N}(k, x)$, in particular for $u=\hat{u}$. This implies

$$
\tilde{J}_{N}(k, x, \hat{u}) \leq \tilde{V}_{N}(k, x)+\tilde{R}_{8}(k, x, M, N) \leq \tilde{J}_{N}(k, x, \hat{u})+\tilde{R}_{8}(k, x, M, N)
$$

and thus $\tilde{R}_{8}(k, x, M, N) \geq 0$. In addition, we know that

$$
\tilde{V}_{N}(k, x) \leq \tilde{J}_{N}(k, x, \hat{u}) \leq \tilde{V}_{N}(k, x)+\tilde{R}_{8}(k, x, M, N) .
$$

With this we can conclude the existence of $R_{8}$ with $\left|R_{8}(k, x, M, N)\right| \leq \tilde{R}_{8}(k, x, M, N)$ such that

$$
\tilde{J}_{N}(k, x, \hat{u})=\tilde{V}_{N}(k, x)+R_{8}(k, x, M, N) .
$$

This finishes the proof.

Using the results from Lemmas $1-3$ we can now prove that the modified optimal value function $\tilde{V}_{N}$ is a Lyapunov function for the system controlled by the MPC feedback $\mu_{N}$ obtained by solving the original (unmodified) MPC problem.

Theorem 4 ( $\tilde{V}_{N}$ Lyapunov function for MPC with unmodified cost). Let Assumptions 1 - 8 hold. Then for each $\Theta>0$ there exists $\delta_{1} \in \mathcal{L}$ such that the optimal value function $\tilde{V}_{N}$ is a Lyapunov function for the closed loop system $g(k, x)=f\left(k, x, \mu_{N}(k, x)\right)$ on $S(k)=Y(k) \backslash \mathbb{P}(k)$ for the families of forward invariant sets $Y(k)=\tilde{V}_{N}^{-1}(k,[0, \Theta])$ and $\mathbb{P}(k)=\tilde{V}_{N}^{-1}\left(k,\left[0, \delta_{1}(N)\right]\right)$.

Proof. ${ }^{4}$ Let $\Theta>0, k \in \mathbb{N}_{0}$ and $x \in \mathbb{X}(k)$. We first prove the existence of lower and upper bounds for $\tilde{V}_{N}(k, x)$ in inequality (10). To obtain a lower bound observe that from Assumption 4 it follows that

$$
\tilde{\ell}(k, x, u) \geq \alpha\left(|x|_{x^{*}(k)}\right)
$$

for all $(x, u) \in \mathbb{X}(k) \times \mathbb{U}(k, x)$. With this we can estimate

$$
\begin{aligned}
\tilde{V}_{N}(k, x) & =\inf _{u \in \mathbb{U}^{N}(k, x)} \sum_{j=0}^{N-1} \tilde{\ell}\left(k+j, x_{u}(j ; x), u(j)\right) \geq \inf _{u \in \mathbb{U}^{N}(k, x)} \sum_{j=0}^{N-1} \alpha\left(\left|x_{u}(j ; x)\right|_{x^{*}(k+j)}\right) \\
& \geq \alpha\left(|x|_{x^{*}(k)}\right)
\end{aligned}
$$

This yields the lower bound $\alpha_{1}=\alpha$. The upper bound follows from Assumption 7 since $\tilde{V}_{N}\left(k, x^{*}(k)\right)=0$ with $\alpha_{2}=\gamma_{\tilde{V}}$.

Now we turn to the inequality (11). Consider the control sequence $\hat{u} \in \mathbb{U}^{N}(k, x)$ defined in Lemma 3 and let $x^{+}:=x_{\hat{u}}(1, x)$. From the definition of the cost functional we have

$$
\tilde{J}_{N}(k, x, \hat{u})=\tilde{\ell}(k, x, \hat{u})+\tilde{J}_{N-1}\left(k+1, x^{+}, \hat{u}(\cdot+1)\right) .
$$

We can apply Lemma 3 to $\tilde{J}_{N}(k, x, \hat{u})$ because $\hat{u}$ exactly corresponds to the control sequence from the lemma. Furthermore, we can apply the lemma to $\tilde{J}_{N-1}\left(k+1, x^{+}, \hat{u}(\cdot+1)\right)$. The reason for this is that the control sequence $\hat{u}(\cdot+1)$ coincides with the control sequence $u_{N-1, x^{+}}^{*}$ up to time $M-1$. This follows from the dynamic programming principle and the fact that tails of optimal control sequences are again optimal control sequences, cf. [11, Corollary 4.5]. From this we obtain

$$
\tilde{V}_{N}(k, x)+R_{8}(k, x, M, N)=\tilde{\ell}(k, x, \hat{u})+\tilde{V}_{N-1}\left(k+1, x^{+}\right)+R_{8}\left(k+1, x^{+}, M-1, N-1\right)
$$

Using Lemma 2 on the right-hand side of the equation for $k=k+1, x=x^{+}, M=M-1$ and $N=N-1$ we get

$$
\begin{gathered}
\tilde{V}_{N}(k, x)+R_{8}(k, x, M, N)=\tilde{\ell}(k, x, \hat{u})+\tilde{V}_{N}\left(k+1, x^{+}\right)+R_{6}\left(k+1, x^{+}, M-1, N-1\right) \\
+R_{8}\left(k+1, x^{+}, M-1, N-1\right)
\end{gathered}
$$

\footnotetext{
${ }^{4}$ Parts of the proof are analogous to the proof of Proposition 8.32 in [11]
} 
or equivalently

$$
\begin{aligned}
\tilde{V}_{N}\left(k+1, x^{+}\right)= & \tilde{V}_{N}(k, x)-\tilde{\ell}(k, x, \hat{u})-R_{6}\left(k+1, x^{+}, M-1, N-1\right) \\
& -R_{8}\left(k+1, x^{+}, M-1, N-1\right)+R_{8}(k, x, M, N) .
\end{aligned}
$$

From Lemma 2 and Lemma 3 we obtain a bound for the residuals

$$
\begin{aligned}
& -R_{6}\left(k+1, x^{+}, M-1, N-1\right)-R_{8}\left(k+1, x^{+}, M-1, N-1\right)+R_{8}(k, x, M, N) \\
& \leq\left|R_{6}\left(k+1, x^{+}, M-1, N-1\right)\right|+\left|R_{8}\left(k+1, x^{+}, M-1, N-1\right)\right|+\left|R_{8}(k, x, M, N)\right| \\
& \leq 2 \gamma_{\tilde{V}}(\sigma(P))+2 \gamma_{\lambda}(\sigma(P))+6 \gamma_{\tilde{V}}(\tilde{\sigma}(P))+2 \gamma_{\lambda}(\tilde{\sigma}(P))+4 \gamma_{V}(N-M, \bar{\sigma}(P))
\end{aligned}
$$

which holds for all $M \in\{0, \ldots, N\} \backslash\left\{\overline{\mathcal{Q}}(k, x, P, N) \cup \overline{\mathcal{Q}}\left(k+1, x^{+}, P, N-1\right)\right\}$. Because each of the sets $\overline{\mathcal{Q}}$ contains at most $2 P$ elements we can choose $P=\left\lfloor\frac{N}{8}\right\rfloor$ to guarantee that there is at least one such $M$ satisfying $M \leq \frac{N}{2}$ which implies $N-M \geq \frac{N}{2}$. With this we can find an upper bound $\nu(N)$ of (35) only depending on $N$ that is given by

$$
\nu(N):=8 \gamma_{\tilde{V}}\left(\sigma\left(\left\lfloor\frac{N}{8}\right\rfloor\right)\right)+2 \gamma_{\lambda}\left(\sigma\left(\left\lfloor\frac{N}{8}\right\rfloor\right)\right)+2 \gamma_{\lambda}\left(\tilde{\sigma}\left(\left\lfloor\frac{N}{8}\right\rfloor\right)\right)+4 \gamma_{V}\left(\frac{N}{2}, \bar{\sigma}\left(\left\lfloor\frac{N}{8}\right\rfloor\right)\right)
$$

using the properties of comparison functions. Thus we arrive at the inequality

$$
\begin{aligned}
\tilde{V}_{N}\left(k+1, x^{+}\right) & \leq \tilde{V}_{N}(k, x)-\tilde{\ell}(k, x, \hat{u})+\nu(N) \\
& =\tilde{V}_{N}(k, x)-\tilde{\ell}\left(k, x, \mu_{N}(k, x)\right)+\nu(N) .
\end{aligned}
$$

In addition, from Assumption 4 it follows that

$$
-\tilde{\ell}(k, x, u) \leq-\alpha\left(|x|_{x^{*}(k)}\right)
$$

for all $(x, u) \in \mathbb{X}(k) \times \mathbb{U}(k, x)$, in particular for $u=\mu_{N}(k, x)$. This leads to the inequality

$$
\tilde{V}_{N}\left(k+1, x^{+}\right) \leq \tilde{V}_{N}(k, x)-\alpha\left(|x|_{x^{*}(k)}\right)+\nu(N) .
$$

Since we have upper bound $\tilde{V}_{N}(k, x) \leq \alpha_{2}\left(|x|_{x^{*}(k)}\right)$ we can further estimate

$$
\begin{aligned}
\tilde{V}_{N}\left(k+1, x^{+}\right) & \leq \tilde{V}_{N}(k, x)-\alpha\left(|x|_{x^{*}(k)}\right)+\nu(N) \\
& \leq \tilde{V}_{N}(k, x)-\alpha\left(\alpha_{2}^{-1}\left(\tilde{V}_{N}(k, x)\right)\right)+\nu(N) \\
& \left.=\tilde{V}_{N}(k, x)-\chi\left(\tilde{V}_{N}(k, x)\right)\right)+\nu(N)
\end{aligned}
$$

with $\chi:=\alpha \circ \alpha_{2}^{-1}$. Define $\delta_{1}(N):=\max \left\{\chi^{-1}(2 \nu(N)), \chi^{-1}(\nu(N))+\nu(N)\right\}$ and let $\mathbb{P}(k):=\tilde{V}_{N}^{-1}\left(k,\left[0, \delta_{1}(N)\right]\right)$. Then for $x \in Y(k) \backslash \mathbb{P}(k)$ it holds that

$$
\tilde{V}_{N}(k, x) \geq \delta_{1}(N) \geq \chi^{-1}(2 \nu(N)) .
$$

This implies

$$
\nu(N) \leq \frac{\chi\left(\tilde{V}_{N}(k, x)\right)}{2}
$$

and it follows that

$$
\begin{aligned}
\tilde{V}_{N}\left(k+1, x^{+}\right) & \left.\leq \tilde{V}_{N}(k, x)-\chi\left(\tilde{V}_{N}(k, x)\right)\right)+\nu(N) \\
& \leq \tilde{V}_{N}(k, x)-\frac{\left.\chi\left(\tilde{V}_{N}(k, x)\right)\right)}{2}
\end{aligned}
$$

and using the lower bound $\alpha_{1}\left(|x|_{x^{*}(k)}\right) \leq \tilde{V}_{N}(k, x)$ we get

$$
\tilde{V}_{N}\left(k+1, x^{+}\right) \leq \tilde{V}_{N}(k, x)-\frac{\left.\chi\left(\tilde{V}_{N}(k, x)\right)\right)}{2} \leq \tilde{V}_{N}(k, x)-\frac{\chi\left(\alpha_{1}\left(|x|_{x^{*}}\right)\right)}{2} .
$$

Thus we have shown the inequality (11) with $\alpha_{V}(r)=\frac{\chi\left(\alpha_{1}(r)\right)}{2}$. What remains to be shown is the forward invariance of the sets $Y(k)$ and $\mathbb{P}(k)$. For $x \in Y(k)$ it holds that $\tilde{V}_{N}(k, x) \leq \Theta$. Now consider $x^{+}$for which it holds

$$
\tilde{V}_{N}(k, x) \leq \tilde{V}_{N}(k, x)-\alpha_{V}\left(|x|_{x^{*}}\right)<\tilde{V}_{N}(k, x) \leq \Theta
$$

and thus $x^{+} \in \tilde{V}_{N}^{-1}(k+1,[0, \Theta])=Y(k+1)$. This shows the forward invariance of $Y(k)$.

To prove forward invariance of $\mathbb{P}(k)$ let $x \in \mathbb{P}(k)$ which implies that $\tilde{V}_{N}(k, x) \leq \delta_{1}(N)$. Distinguish two cases: 
1. case: $\chi\left(\tilde{V}_{N}(k, x)\right) \geq \nu(N)$

Here it follows from (36) that

$$
\tilde{V}_{N}\left(k+1, x^{+}\right) \leq \tilde{V}_{N}(k, x)-\chi\left(\tilde{V}_{N}(k, x)\right)+\nu(N) \leq \tilde{V}_{N}(k, x) \leq \delta_{1}(N) .
$$

2. case: $\chi\left(\tilde{V}_{N}(k, x)\right) \leq \nu(N)$

In this case it follows

$$
\begin{aligned}
\tilde{V}_{N}\left(k+1, x^{+}\right) & \leq \tilde{V}_{N}(k, x)-\chi\left(\tilde{V}_{N}(k, x)\right)+\nu(N) \\
& \leq \tilde{V}_{N}(k, x)+\nu(N)<\chi^{-1}(\nu(N))+\nu(N) \leq \delta_{1}(N)
\end{aligned}
$$

In both cases it follows that $x^{+} \in \mathbb{P}(k+1)$ and thus the forward invariance.

Together with Theorem 2, Theorem 4 shows that the MPC closed loop is practically asymptotically stable at the optimal trajectory. In particular, this means that the closed loop trajectory will converge to a neighborhood of the optimal trajectory, whose size tends to 0 as the optimization horizon $N$ tends to infinity. In addition, Theorem 1 ensures that the closed loop trajectory approaches this neighborhood in an approximately optimal way.

\section{Examples}

In this section we present two examples that illustrate the theoretical results from Theorems 1 and 4 .

Example 1 (Scalar system). Consider the scalar control system

$$
x(k+1)=x(k)+u(k)+w(k)
$$

with a time-varying function $w(k)=-2 \sin \left(\frac{k \pi}{12}\right)+a_{k}$ where $\left(a_{k}\right)_{k \in \mathbb{N}_{0}} \subset\left[-\frac{1}{4}, \frac{1}{4}\right]$ is sequence of random numbers. The stage cost is defined as $\ell(k, x, u)=u^{2}$ and we consider state constraints sets $\mathbb{X}(k)$ defined by

$$
\mathbb{X}(k):= \begin{cases}{[-2,2],} & k \in[24 j, 24 j+12)), j \in \mathbb{N}_{0} \\ {\left[-\frac{1}{2}, \frac{1}{2}\right],} & k \in[24 j+12,24(j+1)), j \in \mathbb{N}_{0}\end{cases}
$$

and control constraints $\mathbb{U}(k)=[-3,3], k \in \mathbb{N}_{0}$.

The model is a minimal example already used in [8]. Though simple in nature, it already captures the important features of time-varying dynamics and economic stage cost. At the same time it is not a purely academic example since it can be interpreted as an application from engineering: regard the state $x$ as the mean temperature of a room that is subject to time-varying ambient temperature fluctuations $w$ caused e.g. by the weather. The aim is to keep the temperature within certain comfort bounds $\mathbb{X}(k)$ by controlling the heating and/or cooling $u$. This goal should be achieved using as little control effort as possible (corresponding for example to electricity cost), which is expressed by the economic stage cost function $\ell$ that only penalizes the control effort.

As such it bears resemblance to a slightly more complex model in continuous time treated in [12], which displays the same essential dynamics but has a periodic optimal solution. In contrast the model we consider has a non-periodic optimal solution due to the external excitation $w$ being non-periodic.

The optimal reference, i.e. the trajectory at which the system operates best on the infinite horizon, cannot be computed analytically for this model. In lieu thereof we compute an open loop trajectory on a very long finite horizon.

It was already shown in [10] that the system is strictly dissipative and satisfies the turnpike and continuity assumptions. This means the requirements for applying Theorem 1 and Theorem 4 are met. The convergence and stability results from the two theorems are also confirmed by our numerical simulations performed in Matlab. The source code of the simulations can be found on Bitbucket ${ }^{5}$. In Figure 2 a MPC closed loop solution for a fixed horizon length is shown. It can be observed that the closed loop follows the optimal trajectory. Figure 3 shows that the MPC closed loop cost converges for increasing horizon length (presumably to the cost of an open loop optimal trajectory on the infinite horizon) and the MPC closed loop trajectory also approximates the optimal trajectory $x$.

Example 2 (Simple PDE model). As a second example we investigate a problem involving a partial differential equation (PDE). The example demonstrates that our theorems not only hold for finite but also infinite dimensional systems.

Consider the convection diffusion equation

$$
\begin{gathered}
\frac{\partial y}{\partial t}-\alpha \nabla^{2} y+w \nabla y=0 \text { on } Q:=\Omega \times[0, T] \\
y(0)=y_{0} \text { on } \Omega
\end{gathered}
$$

\footnotetext{
${ }^{5}$ Bitbucket repository: https://bitbucket.org/spirkelmann/scalar-economic-mpc-example/, commit: 55f42af
} 

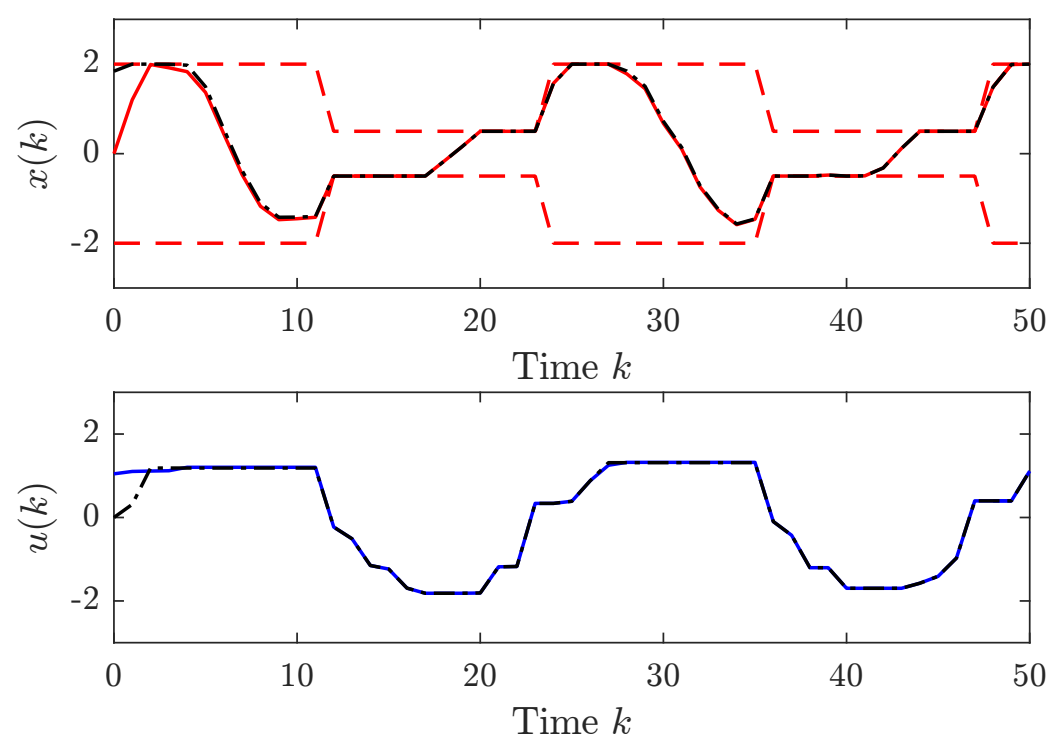

Figure 2: MPC closed loop state (solid red) and control (solid blue) trajectories for horizon length $N=8$ and optimal trajectory (dash-dotted black). The MPC trajectory starts away from the optimal trajectory at $x(0)=0$ and quickly converges to the optimal trajectory.
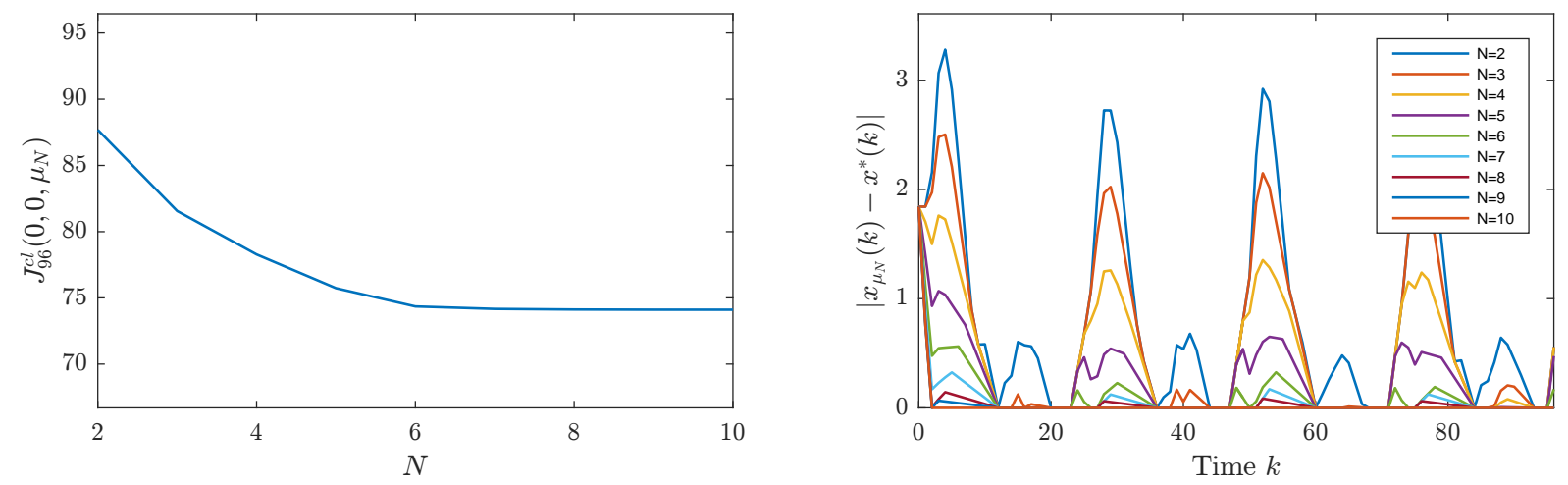

Figure 3: Left: Evolution of the closed loop cost $J_{L}^{c l}\left(k, x, \mu_{N}\right)$ for $L=96$ time steps with increasing the horizon length $N$. Right: Convergence of the MPC closed loop trajectory $x_{\mu_{N}}$ towards the optimal trajectory $x^{*}$ for increasing horizon length. 
where $y: Q \rightarrow \mathbb{R}$ is the temperature, $\alpha>0$ is the diffusion coefficient, $w: \Omega \times[0, T] \rightarrow \mathbb{R}$ is a velocity field and $y_{0}: \Omega \rightarrow \mathbb{R}$ is the initial condition at time zero. Let the boundary of the domain be separated in two parts $\Gamma_{\text {out }}$ and $\Gamma_{c}$ and consider boundary conditions of Robin type:

$$
\begin{gathered}
\frac{\partial y}{\partial n}+\gamma_{\text {out }} y=\delta_{\text {out }} y_{\text {out }} \text { on } \Sigma_{\text {out }}:=\Gamma_{\text {out }} \times[0, T] \\
\frac{\partial y}{\partial n}+\gamma_{c} y=\delta_{c} u \text { on } \Sigma_{c}:=\Gamma_{c} \times[0, T]
\end{gathered}
$$

In the above equations $\frac{\partial y}{\partial n}$ is the derivative of $y$ in normal direction, $y_{\text {out }}: \Sigma_{\text {out }} \rightarrow \mathbb{R}$ is the outside temperature, $u: \Sigma_{c} \rightarrow \mathbb{R}$ is a control function, and $\gamma_{c}, \delta_{c}: \Sigma_{c} \rightarrow \mathbb{R}, \gamma_{\text {out }}, \delta_{\text {out }}: \Sigma_{\text {out }} \rightarrow \mathbb{R}$ are coefficient functions.

The optimal control problem is given by:

$$
\min _{y, u, w} J(y, u, w)=\frac{1}{2}\|u\|_{L^{2}\left(\Sigma_{c}\right)}^{2}+\frac{1}{2}\|w\|_{L^{2}(Q)}^{2}
$$

subject to equations (37), (38) and the constraints

$$
\begin{aligned}
& \underline{u} \leq u \leq \bar{u} \text { on } \Sigma_{c} \\
& \underline{y} \leq y \leq \bar{y} \text { on } \Omega_{y} \times[0, T]
\end{aligned}
$$

with lower and upper bounds for state and control where $\Omega_{y} \subseteq \Omega$ is a subdomain.

The physical interpretation of this setting is similar to Example 1 but now with an underlying PDE. The state $y$ models the spatial distribution of the temperature within a room. The temperature is subject to time-dependent variations at the boundary $\Gamma_{\text {out }}$ due to changing outside temperature $y_{\text {out }}$. On the controlled part of the boundary $\Gamma_{c}$ the temperature can be influenced by the control $u$ representing heating and cooling. In addition, a second control $w$ can be used to affect the convection, similar to a controllable air flow inside the room. The goal is to keep the temperature of the room within lower and upper bounds $\underline{y}$ and $\bar{y}$ on the subdomain $\Omega_{y}$, using as little energy as possible.

For simplicity we consider the unit interval as domain $\Omega$. Similar results can be obtained also in higher dimensions, see also [13]. The boundary $\Gamma$ is partitioned into an uncontrolled boundary $\Gamma_{\text {out }}$ at $x=0$ and a controlled boundary $\Gamma_{c}$ at $x=1$, see Figure 4. Furthermore, we assume the controlled convection $w$ is constant in space. For the solution with MPC the problem is discretized using linear Lagrange finite elements for

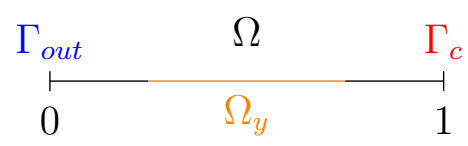

Figure 4: Illustration of domain $\Omega$ and subdomain $\Omega_{y}$, as well as controlled $\left(\Gamma_{c}\right)$ and uncontrolled $\left(\Gamma_{\text {out }}\right)$ parts of the boundary.

the spatial discretization and the implicit Euler method for the time discretization. The numerical simulations have been carried out in Python with the help of FEniCS [14] [15] and Ipopt [16]. All the parameters for the simulations can be found in Table 1. The source code for the simulations can be found on Bitbucket ${ }^{6}$.

\begin{tabular}{|c|c|c||c|c|c|}
\hline Parameter & Value & Description & Parameter & Value & Description \\
\hline$\Omega_{y}$ & {$[0.25,0.75]$} & subdomain & $\alpha$ & 1 & diffusion coefficient \\
$h$ & 0.01 & sampling rate & $\gamma_{\text {out }}, \delta_{\text {out }}$ & $10^{6}$ & parameters at outside boundary \\
$n_{y}$ & 100 & dof for FEM discretization & $\gamma_{c}$ & 0 & parameter at control boundary \\
$-\underline{y}, \bar{y}$ & 0.15 & state constraints & $\delta_{c}$ & 10 & parameter at control boundary \\
$-\underline{u}, \bar{u}$ & 0.25 & control constraints & $y_{\text {out }}(t)$ & $0.3 \sin (10 t)$ & time-varying outside temperature \\
$y_{0}$ & -0.1 & initial value of the state & & & \\
\hline
\end{tabular}

Table 1: Overview of parameters used in the simulations.

It seems difficult to verify the turnpike assumption analytically for this example. However, numerical evidence from Figure 5 suggests that the problem has the turnpike property. Like in Example 1 the optimal trajectory has been computed from an optimization with a free initial value on a long horizon. The plots indicate that open loop trajectory come close to the turnpike, i.e. the optimal trajectory $y^{*}$. It can also be observed that for a longer

\footnotetext{
${ }^{6}$ Bitbucket repository: https://bitbucket.org/spirkelmann/pde-economic-mpc-example/, commit: aebbf8a
} 

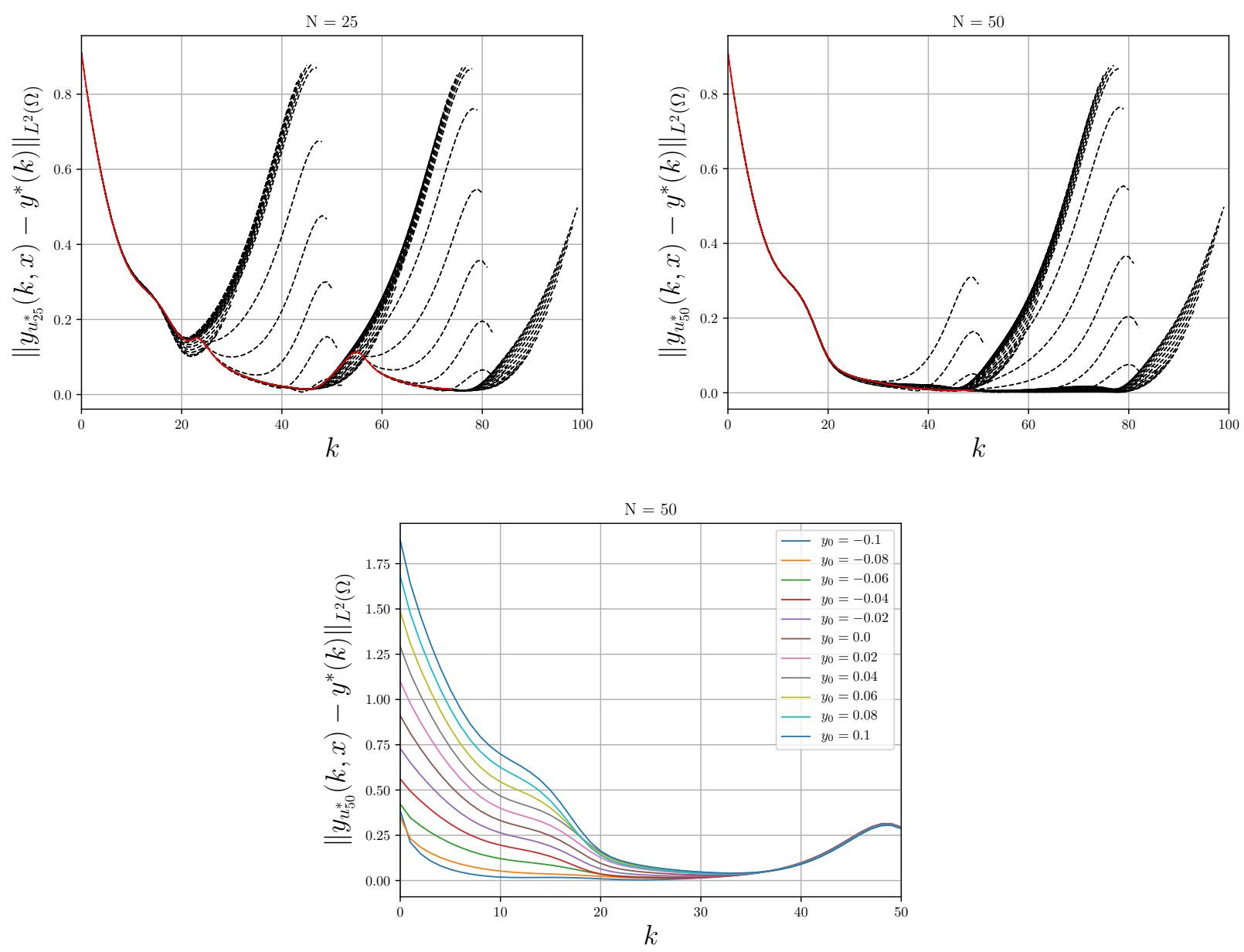

Figure 5: The upper two figures show the norm difference $\left\|y_{u_{N}^{*}}(k, x)-y^{*}(k)\right\|_{L^{2}(\Omega)}$ between open loop predictions $y_{u_{N}^{*}}$ starting at time $k$ with initial values on the closed loop $y_{\mu_{N}}(k)$ and the optimal trajectory $y^{*}$ (dashed black line) for horizon length $N=25$ on the left and $N=50$ on the right. In addition the norm difference of the closed loop $y_{\mu_{N}}$ to the optimal trajectory $y^{*}$ is shown (solid red line). The plot at the bottom shows the norm difference between open loop predictions $y_{u_{N}^{*}}$ and the optimal trajectory $y^{*}$ starting at different initial values $y_{0}$ for a fixed horizon length $N=50$.

horizon the open loop solutions stay longer at the turnpike which also causes the closed loop to be closer to the optimal trajectory. Moreover, the third plot in Figure 5 shows that even for different initial values the open loop predictions are at some point near the optimal trajectory, although for some initial values this happens faster than for others.

Checking the continuity assumptions of the optimal value functions is more involved and remains an open problem. Nonetheless we can apply the MPC algorithm to the problem. Figure 6 shows an exemplary MPC closed loop with a horizon of $N=50$. The state on the subdomain $\Omega_{y}$ is kept between the lower and upper bounds. The control $u$ alternates between cooling and heating in order to counteract the rising and falling temperature at the uncontrolled boundary. As seen in Figure 7 we can observe convergence of the closed loop cost of the MPC solutions for increasing horizon length as well as convergence of the MPC closed loop trajectories to the optimal trajectory. An interesting observation is that in this example the cost converges much quicker than the state. While there is already not much change in the value of the closed loop cost for $N=20$ the closed loop trajectories themselves only come close to the optimal trajectory around relatively long horizons of $N=70$.

\section{Conclusions}

In this paper we presented performance and stability results for economic model predictive control in the timevarying setting. We generalized the concept of optimal equilibria and optimal periodic orbits to the time-varying setting, making use of a overtaking optimality as a more general optimality notion. This resulted in the definition of an optimal trajectory. We then showed that under turnpike and continuity assumptions the cost of the MPC closed loop approximates the cost of optimal trajectories on the infinite horizon. In addition, we used strict 


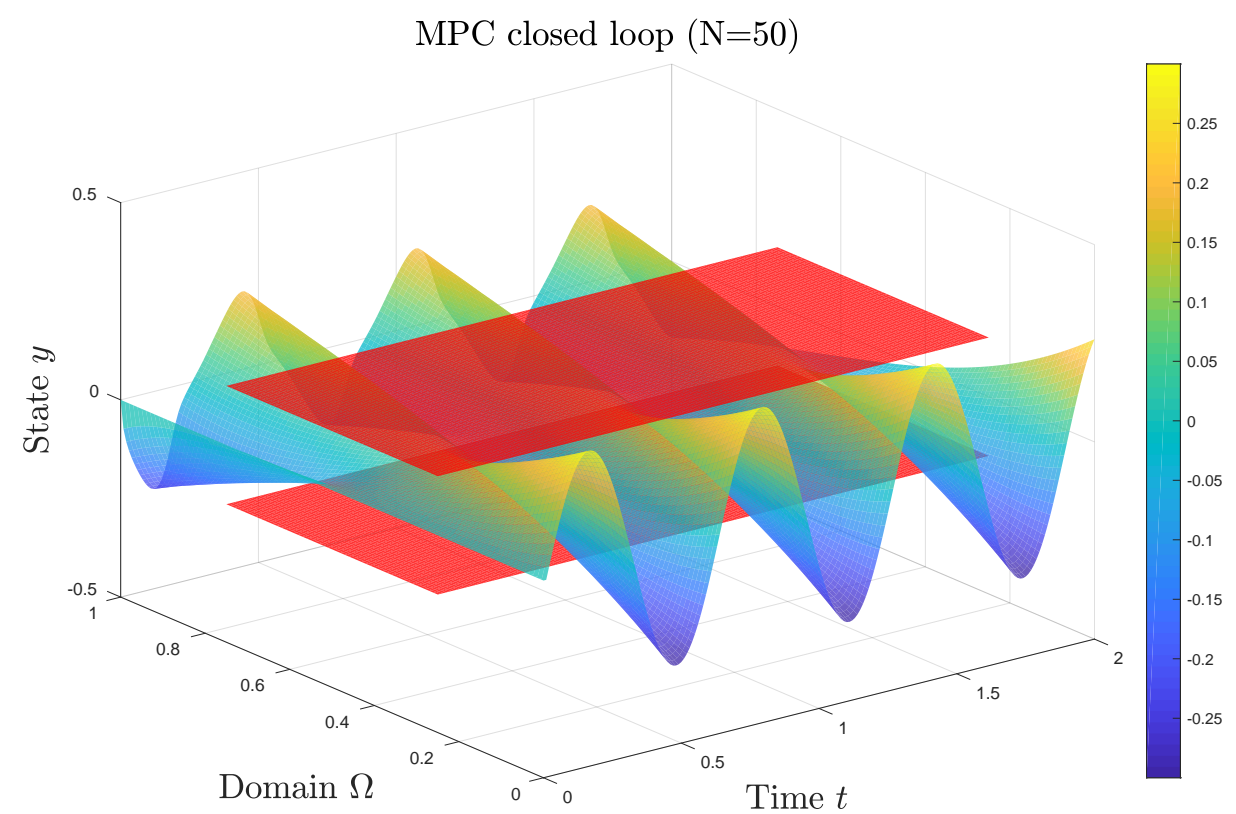

Figure 6: Temporal and spatial evolution of an MPC closed loop trajectory for horizon length $N=50$. The state constraints on the subdomain $\Omega_{y}=[0.25,0.75]$ are plotted in red.

dissipativity in order to prove that a modified optimal value function is a practical Lyapunov function, allowing us to conclude that the MPC closed loop converges to a neighborhood of the optimal trajectory whose size tends to 0 as the optimization horizon $N$ tends to infinity. Finally, we presented two examples that illustrated that our theoretical findings also hold in practical applications, both for finite and infinite dimensional systems. 

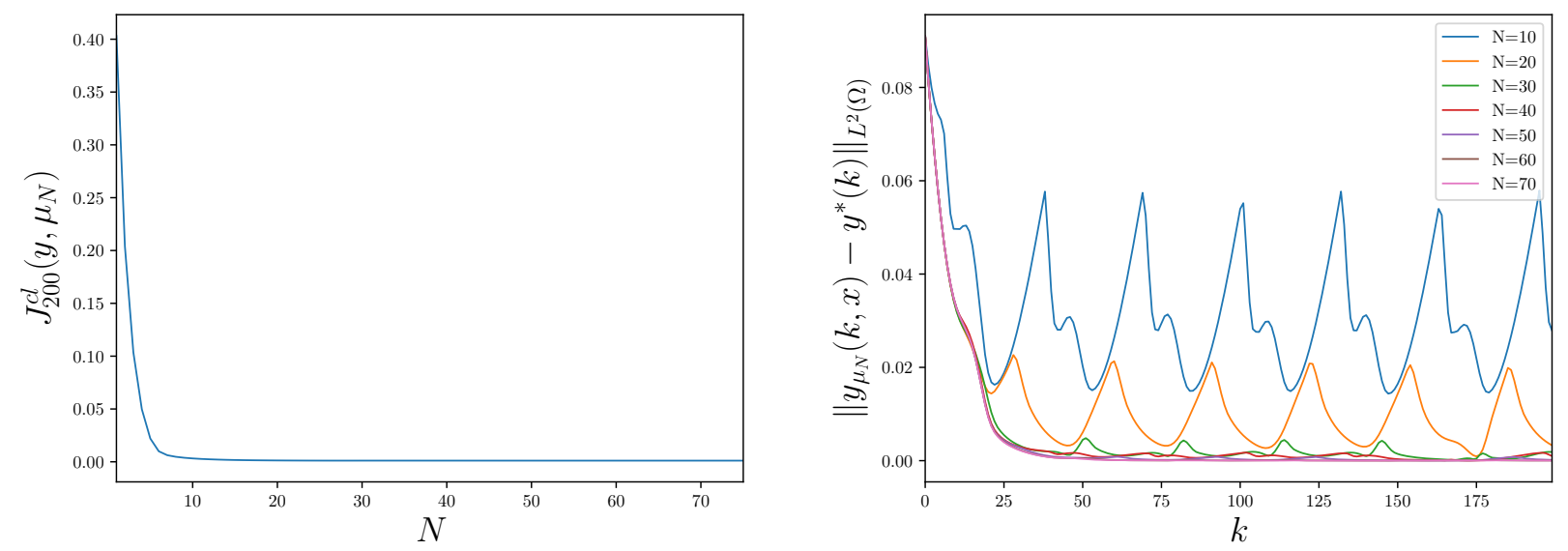

Figure 7: Left: Evolution of the closed loop cost $J_{200}^{c l}\left(y, \mu_{N}\right)$ for $L=200$ time steps with different horizon lengths $N$. Right: Norm difference $\left\|y_{\mu_{N}}(k, x)-y^{*}(k)\right\|_{L^{2}(\Omega)}$ between MPC closed loop trajectories and the optimal trajectory at each time point for different horizon lengths $N$.

\section{Financial disclosure}

This research was supported by DFG-Grant GR 1569/16-1.

\section{Conflict of interest}

The authors declare no potential conflict of interests.

\section{References}

[1] Rishi Amrit, James B. Rawlings, and David Angeli. Economic optimization using model predictive control with a terminal cost. Annual Rev. Control, 35:178-186, 2011.

[2] Lars Grüne. Economic receding horizon control without terminal constraints. Automatica, 49(3):725-734, 2013.

[3] Matthias A. Müller and Lars Grüne. Economic model predictive control without terminal constraints for optimal periodic behavior. Automatica, 70:128-139, August 2016.

[4] Timm Faulwasser, Lars Grüne, and Matthias A. Müller. Economic nonlinear model predictive control. Foundations and Trends in Systems and Control, 5(1):1-98, January 2018.

[5] Mario Zanon, Sebastien Gros, and Moritz Diehl. A Lyapunov function for periodic economic optimizing model predictive control. In, editor, Proceedings of the 52nd IEEE Conference on Decision and Control (CDC2013), pages 5107-5112, Florence, Italy, 2013.

[6] Mario Zanon, Lars Grüne, and Moritz Diehl. Periodic optimal control, dissipativity and MPC. IEEE Trans. Autom. Control, 62(6):2943-2949, 2017.

[7] Julian Berberich. Indefinite linear quadratic optimal control: periodic dissipativity and turnpike properties. Master's thesis, . Institute for Systems Theory and Automatic Control, University of Stuttgart;, 22018.

[8] Lars Grüne and Simon Pirkelmann. Closed-loop performance analysis for economic model predictive control of time-varying systems. In, editor, Proceedings of the 56th IEEE Conference on Decision and Control (CDC 2017), pages 5563-5569, Dec 2017.

[9] David Gale. On optimal development in a multi-sector economy. The Review of Economic Studies, 34(1):1$18,1967$.

[10] Lars Grüne, Simon Pirkelmann, and Marleen Stieler. Strict dissipativity implies turnpike behavior for time-varying discrete time optimal control problems. In Gustav Feichtinger, Raimund M. Kovacevic, and Gernot Tragler, editors, Control Systems and Mathematical Methods in Economics: Essays in Honor of Vladimir M. Veliov, pages 195-218, Cham, 2018. Springer International Publishing. 
[11] Lars Grüne and Jürgen Pannek. Nonlinear Model Predictive Control. Theory and Algorithms. Springer, second edition, 2017.

[12] James B. Rawlings and Michael J. Risbeck. Model predictive control with discrete actuators: Theory and application. Automatica, 78:258 - 265, 2017.

[13] Luca Mechelli and Stefan Volkwein. Pod-based economic model predictive control for heat-convection phenomena. In Florin Adrian Radu, Kundan Kumar, Inga Berre, Jan Martin Nordbotten, Iuliu Sorin Pop , editor, Numerical Mathematics and Advanced Applications - ENUMATH 2017, page To appear, Dec 2018.

[14] Martin S. Alnæs, Jan Blechta, Johan Hake, August Johansson, Benjamin Kehlet, Anders Logg, Chris Richardson, Johannes Ring, Marie E. Rognes, and Garth N. Wells. The fenics project version 1.5. Archive of Numerical Software, 3(100), 2015.

[15] Anders Logg, Kent-Andre Mardal, and Garth N. Wells, editors. Automated Solution of Differential Equations by the Finite Element Method. The FEniCS Book. Springer, 2012.

[16] Andreas Wächter and Lorenz T. Biegler. On the implementation of an interior-point filter line-search algorithm for large-scale nonlinear programming. Mathematical Programming, 106(1):25-57, Mar 2006.

[17] David Angeli, Rishi Amrit, and James B. Rawlings. On average performance and stability of economic model predictive control. IEEE Trans. Autom. Control, 57(7):1615-1626, 2012.

[18] David Angeli, Alessandro Casavola, and Francesco Tedesco. Theoretical advances on economic model predictive control with time-varying costs. Annual Reviews in Control, 41:218-224, 2016.

[19] Joël Blot and Naïla Hayek. Infinite-horizon optimal control in the discrete-time framework. Springer, 2014.

[20] Lars Grüne. Approximation properties of receding horizon optimal control. Jahresbericht der Deutschen Mathematiker-Vereinigung, 118(1):3-37, 2016.

[21] Lars Grüne and Marleen Stieler. Asymptotic stability and transient optimality of economic mpc without terminal conditions. Journal of Process Control, 24(8):1187-1196, 2014.

[22] Christopher M. Kellett. A compendium of comparison function results. Mathematics of Control, Signals, and Systems, 26(3):339-374, 2014. 